\title{
Solutions for the Stefan problem with Gibbs-Thomson law by a local minimisation
}

\author{
MATTHiAS RÖGER ${ }^{\dagger}$ \\ Institut für Angewandte Mathematik, Universität Bonn, \\ Wegelerstr. 6, 53115 Bonn, Germany
}

[Received 16 May 2003 and in revised form 22 October 2003]

\begin{abstract}
A new construction scheme for a time-discrete version of the Stefan problem with Gibbs-Thomson law is introduced. Extending a scheme due to Luckhaus [11] our approach uses a local minimisation of certain penalised functionals instead of minimising these functionals globally. The main difference is that local minimisation allows for surface loss of approximate phase interfaces in the limit. The theory of varifolds is used to obtain the convergence of approximate Gibbs-Thomson equations. A particular situation exhibits that local minimisation provides more physically appealing solutions than those constructed by global minimisation.
\end{abstract}

\section{Introduction}

The Stefan problem and its variants describe phase transitions like melting or solidification of a given material. The Gibbs-Thomson law accounts for surface tension effects and introduces a geometric condition on the phase boundary. This generalisation of the classical Stefan problem allows modelling phenomena like superheating or undercooling of phases. For a derivation of the model see [9] and [22].

Let a time interval $(0, T)$ and an open bounded region $\Omega \subset \mathbb{R}^{3}$ with Lipschitz boundary be given, and set $\Omega_{T}:=(0, T) \times \Omega$. We are looking for a phase and a temperature function,

$$
\mathcal{X}: \Omega_{T} \rightarrow\{0,1\} \quad \text { and } \quad u: \Omega_{T} \rightarrow \mathbb{R} .
$$

Here the set $\{\mathcal{X}(t)=1\}$ represents the liquid phase, $\{\mathcal{X}(t)=0\}$ the solid phase. The common boundary in $\Omega$ describes the phase interface. The governing equations are the energy balance

$$
\partial_{t}(u+\mathcal{X})-\Delta u=f
$$

and the Gibbs-Thomson law on the phase interface

$$
H(t, \cdot)=u(t, \cdot)
$$

Here $f$ is a given heat source and $H(t, \cdot)$ denotes the mean curvature of the phase interface at time $t \in(0, T)$. Mean curvature is taken positive for convex liquid phases. We impose an initial condition for $u+\mathcal{X}$ and boundary conditions for $u$, namely we assume that on a subset $\Gamma_{\mathrm{D}}$ of $\partial \Omega$ with positive

\footnotetext{
${ }^{\dagger}$ M. Röger was supported by DFG Sonderforschungsbereich 611 and by the European Community’s Human Potential Programme under contract HPRN-CT-2002-00274, FRONTS-SINGULARITIES.

Email: roeger@iam.uni-bonn.de
} 
$\mathcal{H}^{2}$-measure a Dirichlet condition is prescribed. In [11] (see also [10]) Luckhaus gives a first long time existence result for weak solutions. He proves the existence of functions

$$
\mathcal{X} \in \mathrm{L}^{\infty}(0, T ; \operatorname{BV}(\Omega ;\{0,1\})), \quad u \in \mathrm{L}^{2}\left(0, T ; \mathrm{H}^{1,2}(\Omega)\right),
$$

solving $(1.1)$ in the sense of distributions and $[1.2$ in the following $B V$-formulation of the GibbsThomson law:

$$
\int_{0}^{T} \int_{\Omega}\left(\nabla \cdot \xi-\frac{\nabla \mathcal{X}}{|\nabla \mathcal{X}|} \cdot D \xi \frac{\nabla \mathcal{X}}{|\nabla \mathcal{X}|}\right)(t, \cdot)|\nabla \mathcal{X}|(t, \cdot) \mathrm{d} t=\int_{\Omega_{T}} \nabla \cdot(u \xi) \mathcal{X}
$$

for all $\xi \in C_{\mathrm{c}}^{\infty}\left(\Omega_{T} ; \mathbb{R}^{3}\right)$. In [11] an implicit time-discretisation is used and approximate phase functions are chosen as global minimisers of appropriate functionals. This global minimisation preserves the total surface area of the phase interfaces when the time-steps approach zero. As a consequence approximate Gibbs-Thomson laws converge within the BV-formulation and in the limit phase functions enjoy additional minimising and regularity properties. Singularities of phase boundaries, like cusps, which for example can arise when two parts of one phase merge, are excluded. In this sense solutions constructed by global minimisation are too restrictive. In [13] Plotnikov and Starovortov proved that solutions of a certain phase field model converge to weak solutions of the Stefan problem with Gibbs-Thomson law. The authors use the same notion of weak solutions which enjoy the same minimising and regularity properties as those of [11].

In the present paper, as an alternative to global minimisation, a local minimisation is used to construct approximate phase functions. We use the same time-discrete functionals as used in [11] but introduce a second discrete evolution. Approximate phase functions are chosen as suitable stationary points instead of global minimisers. Our construction admits quite general phase interfaces and provides more physically appealing solutions than those constructed by global minimisation.

From a technical point of view the main difference to global minimisation is that local minimisation allows a loss of total surface mass for the phase interfaces when passing to the limit with time-discrete approximations. The following time-independent example presents some difficulties which arise. Consider approximate phase functions $\mathcal{X}^{h}$ each consisting of two solid parts and let the solid parts merge with $h \rightarrow 0$.
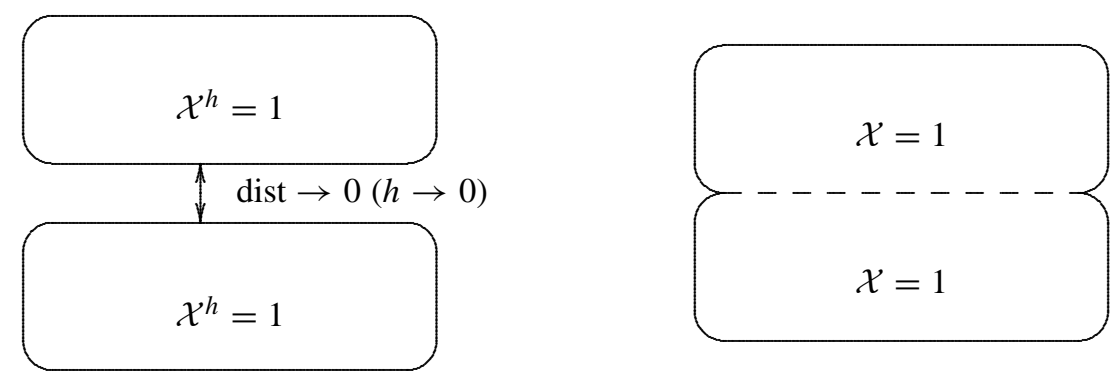

A part of the boundary, indicated by the dashed line, has ceased to separate two different phases. We call this part the hidden boundary, whereas the phase interface represents the physically relevant part of the boundary. Cusp singularities occur due to the cancellation of phase interfaces. As shown in [16] the BV-formulation of the Gibbs-Thomson law breaks down. Thus a more general formulation has to be given. 
Following an idea from [17] we deal with the surface measure of the phase boundaries. In the above example the limit $h \rightarrow 0$ yields a Radon measure with double multiplicity on the hidden boundary. To treat such objects we use the concept of integral varifolds as introduced by Almgren [1]. In Geometric Measure Theory a notion of mean curvature for varifolds has been developed. Schätzle [17] investigates the limit behaviour of surfaces with mean curvature given by a Sobolev function in the ambient space. In this context it is useful to take hidden boundaries into account. On the other hand the Gibbs-Thomson law is relevant only on the phase interface. In Section 3 we justify the following generalised notion of mean curvature for quite general phase interfaces.

Definition 1.1 Let $\Omega \subset \mathbb{R}^{n}$ be open, $E \subset \Omega$ and $\mathcal{X}_{E} \in \mathrm{BV}(\Omega)$. Assume there is an integral $(n-1)$-varifold $\mu$ on $\Omega$ such that

$$
\partial^{*} E \subset \operatorname{spt}(\mu)
$$

and $\mu$ has locally bounded first variation with mean curvature vector $\vec{H}_{\mu}$ satisfying

$$
\vec{H}_{\mu} \in \mathrm{L}_{\mathrm{loc}}^{s}(\mu), \quad s>n-1, s \geqslant 2 .
$$

Then we call

$$
\vec{H}=\left.\vec{H}_{\mu}\right|_{\partial^{*} E}
$$

the generalised mean curvature vector of $\partial^{*} E$.

We use this definition to extend the BV-formulation of the Gibbs-Thomson law. The main result of this paper is the following existence theorem.

THEOREM 1.2 Let $T>0$ and $\Omega \subset \mathbb{R}^{3}$ be an open bounded set with Lipschitz boundary, assume $\Gamma_{\mathrm{D}} \subset \partial \Omega$ with $\mathcal{H}^{2}\left(\Gamma_{\mathrm{D}}\right)>0$ and define

$$
M_{0}=\left\{v \in \mathrm{H}^{1,2}(\Omega):\left.v\right|_{\Gamma_{\mathrm{D}}}=0\right\} .
$$

For given data

$$
\begin{aligned}
u_{0} & \in \mathrm{L}^{\infty}(\Omega) \cap \mathrm{H}^{1,2}(\Omega), \\
\mathcal{X}_{0} & \in \mathrm{BV}(\Omega ;\{0,1\}), \\
u_{\mathrm{D}} & \in \mathrm{H}^{1,2}(\Omega) \cap \mathrm{L}^{\infty}(\Omega), \\
f & \in \mathrm{L}^{\infty}(\Omega),
\end{aligned}
$$

there exist functions

$$
\begin{gathered}
\mathcal{X} \in \mathrm{L}^{\infty}(0, T ; \operatorname{BV}(\Omega ;\{0,1\})), \\
u \in \mathrm{L}^{2}\left(0, T ; u_{\mathrm{D}}+M_{0}\right) \cap \mathrm{L}^{\infty}\left(0, T ; \mathrm{L}^{p}(\Omega)\right) \quad \text { for all } 1 \leqslant p<\infty,
\end{gathered}
$$

constructed by a local minimisation, such that

$$
\int_{\Omega_{T}}(u+\mathcal{X}) \partial_{t} \varphi+\int_{\Omega}\left(u_{0}+\mathcal{X}_{0}\right) \varphi(0)-\int_{\Omega_{T}} \nabla u \cdot \nabla \varphi=-\int_{\Omega_{T}} f \varphi
$$


for all $\varphi \in C_{\mathrm{c}}^{\infty}([0, T) \times \bar{\Omega})$ with $\varphi=0$ on $\Gamma_{\mathrm{D}}$. Moreover for almost all $t \in(0, T)$ the reduced boundary of $E(t)=\{\mathcal{X}(t, \cdot)=1\}$ has a generalised mean curvature vector $\vec{H}(t)$, which $\mathcal{H}^{2}$-almost everywhere on $\partial^{*} E(t)$ satisfies the Gibbs-Thomson law

$$
\vec{H}(t)=u(t) v(t)
$$

where $v(t)=\nabla \mathcal{X}(t) /|\nabla \mathcal{X}(t)|$ on $\partial^{*} E(t)$.

The proof of the theorem is given in Sections 4 and 5 Section 6 investigates the behaviour of solutions. We study a particular situation where two ice balls grow monotonically. Solutions constructed by global minimisation build a bridge-like connection at a positive distance whereas solutions by local minimisation can touch continuously.

Acknowledgements. This paper originates from the doctoral thesis of the author [15]. I thank my advisers H. W. Alt and R. Schätzle for many fruitful discussions and their constant support.

\section{General definitions and notation}

We fix some notations and recall some basic definitions. As a general reference for Geometric Measure Theory see the book of Simon [19].

For functions depending on time and space variables denote by " $\nabla$ " and " $\nabla$." the spatial gradient and spatial divergence, respectively. For a differentiable function $f: \mathbb{R}^{n} \rightarrow \mathbb{R}^{n}$ and a $k$-dimensional subspace $T$ of $\mathbb{R}^{n}$ we define the divergence restricted to $T$ by

$$
\operatorname{div}_{T} f(x):=\sum_{i=1}^{k} t_{i} \cdot D f(x) t_{i},
$$

where $\left\{t_{i}\right\}_{i=1, \ldots, k}$ is any orthonormal basis of $T$.

Let $\omega_{k}$ denote the volume of the $k$-dimensional unit ball and define for $(y, t) \in \mathbb{R}^{n-1} \times \mathbb{R}$ and $\varrho, \sigma>0$ the cylinder

$$
Z_{\varrho, \sigma}((y, t)):=B_{\varrho}^{n-1}(y) \times(t-\sigma, t+\sigma) .
$$

Let $\Omega \subset \mathbb{R}^{n}$ be open and $\mu$ a Radon measure on $\Omega$. We define the $k$-dimensional density of $\mu$ at a point $x \in \Omega$,

$$
\theta^{k}(\mu, x):=\lim _{\varrho \downarrow 0} \frac{\mu\left(B_{\varrho}(x)\right)}{\omega_{k} \varrho^{k}},
$$

and for a subset $A \subset \Omega$ the density

$$
\theta(\mu, A, x):=\lim _{\varrho \downarrow 0} \frac{\mu\left(B_{\varrho}(x) \cap A\right)}{\mu\left(B_{\varrho}(x)\right)},
$$

if the respective limits exist. If $\theta(\mu, A, x)=1$ we say that the set $A$ has full density at $x$ (with respect to $\mu$ ).

For $x \in \Omega$ and $\varrho>0$ define the scaled measures

$$
\mu_{x, \varrho}(A):=\varrho^{-n+1} \mu(x+\varrho A) .
$$


A $k$-dimensional subspace $P \subset \mathbb{R}^{n}$ is called the $k$-dimensional tangential plane of $\mu$ at $x$, denoted by $T_{x} \mu$, if there is $\theta>0$ such that

$$
\mu_{x, \varrho} \rightarrow \theta \mathcal{H}^{k}\llcorner P \quad \text { as Radon measures }
$$

as $\varrho$ goes to zero. In this case $\theta$ is the multiplicity of $\mu$ in $x$.

We call $\mu$ a rectifiable $(n-1)$-varifold if for $\mu$-almost all $x \in \Omega$ the $(n-1)$-dimensional tangential plane $T_{x} \mu$ exists, and an integral $(n-1)$-varifold if in addition $\theta^{n-1}(\mu, \cdot)$ is $\mu$-almost everywhere integer-valued. A general $(n-1)$-varifold is a Radon measure on the Grassmannian $G^{n-1} \Omega$, which is the product of $\Omega$ and the space of $(n-1)$-dimensional unoriented subspaces of $\mathbb{R}^{n}$. In the present paper we identify a rectifiable $(n-1)$-varifold $\mu$ and the related Radon measure $V_{\mu}$ on $G^{n-1} \Omega$, defined by

$$
V_{\mu}(\zeta):=\int_{\Omega} \zeta\left(x, T_{x} \mu\right) \mathrm{d} \mu(x) \quad \text { for } \zeta \in C_{\mathrm{c}}^{0}\left(G^{n-1} \Omega\right) .
$$

The first variation of a rectifiable $(n-1)$-varifold $\mu$ is given by

$$
\delta \mu(\xi):=\int_{\Omega} \operatorname{div}_{T_{x} \mu} \xi(x) \mathrm{d} \mu(x) \quad \text { for } \xi \in C_{\mathrm{c}}^{1}\left(\Omega ; \mathbb{R}^{n}\right) .
$$

We say $\mu$ is of locally bounded first variation with mean curvature vector $\vec{H}_{\mu}$ if $\vec{H}_{\mu} \in \mathrm{L}_{\mathrm{loc}}^{1}(\mu)$ and

$$
\delta \mu(\xi)=\int_{\Omega}-\vec{H}_{\mu} \cdot \xi \mathrm{d} \mu \quad \text { for all } \xi \in C_{\mathrm{c}}^{1}\left(\Omega ; \mathbb{R}^{n}\right) .
$$

A point $x_{0} \in \operatorname{spt}(\mu)$ is called generic with respect to $\mu$ if the following conditions hold:

- the tangential plane $T_{x_{0}} \mu$ exists,

- $\theta^{n-1}\left(\mu, x_{0}\right)=\theta_{0} \in \mathbb{N}$,

- $\theta\left(\mu,\left\{\theta^{n-1}(\mu, \cdot)=\theta_{0}\right\}, x_{0}\right)=1$.

For $A \subset \Omega, x_{0} \in \Omega$ and $\varphi: A \rightarrow \mathbb{R}^{m}$, we call $y \in \mathbb{R}^{m}$ the approximate limit of $\varphi$ at $x_{0}$ if for all $\varepsilon>0$,

$$
\theta^{n}\left(\Omega \backslash\{|\varphi(\cdot)-y|<\varepsilon\}, x_{0}\right)=0 .
$$

In this case we write $y=\operatorname{ap}-\lim _{x \rightarrow x_{0}} \varphi(x)$.

A function $\varphi: A \rightarrow \mathbb{R}$ is twice approximately differentiable at $x_{0} \in \Omega$ if there are a vector $b \in \mathbb{R}^{n}$ and a symmetric matrix $S \in \mathbb{R}^{n \times n}$ such that

$$
\operatorname{ap}_{x \rightarrow x_{0}} \frac{\left|\varphi(x)-\varphi\left(x_{0}\right)-b \cdot\left(x-x_{0}\right)-\frac{1}{2}\left(x-x_{0}\right)^{T} S\left(x-x_{0}\right)\right|}{\left|x-x_{0}\right|^{2}}=0 .
$$

Then we set

$$
\nabla \varphi\left(x_{0}\right)=b, \quad D^{2} \varphi\left(x_{0}\right)=S .
$$

For a $\mathcal{L}^{n}$-measurable set $E \subset \Omega$ of finite perimeter let $\partial^{*} E$ denote the reduced boundary of $E$ in $\Omega$, that is, the subset of $\partial E \cap \Omega$ where a generalised inner normal exists as Radon-Nikodym derivative $\nabla \mathcal{X}_{E} /\left|\nabla \mathcal{X}_{E}\right|$ with length one. Then $\left|\nabla \mathcal{X}_{E}\right|=\mathcal{H}^{n-1}\left\llcorner\partial^{*} E\right.$ is an integral (with density 1) $(n-1)$-varifold on $\Omega$ (see [3, Section 3.5]). 


\section{A notion of mean curvature for general phase interfaces}

To give an extension of the Gibbs-Thomson law we justify the notion of generalised mean curvature given in Definition 1.1 We are concerned with phase interfaces given as boundaries of Caccioppoli sets that can be completed to integral varifolds. If the latter have a mean curvature of sufficiently high integrability, we prove that on the phase interface this mean curvature is independent of the completion.

Proposition 3.1 Let $\Omega \subset \mathbb{R}^{n}$ be open, $E \subset \Omega$ and $\mathcal{X}_{E} \in \mathrm{BV}(\Omega)$. Assume that there are integral $(n-1)$-varifolds $\mu_{1}, \mu_{2}$ on $\Omega$ such that for $i=1,2$,

$$
\partial^{*} E \subset \operatorname{spt}\left(\mu_{i}\right)
$$

$\mu_{i}$ is of locally bounded first variation with mean curvature vector $\vec{H}_{\mu_{i}}$, and

$$
\vec{H}_{\mu_{i}} \in \mathrm{L}_{\mathrm{loc}}^{s}\left(\mu_{i}\right), \quad s>n-1, s \geqslant 2 .
$$

Then

$$
\vec{H}_{\mu_{1}}=\vec{H}_{\mu_{2}} \quad \mathcal{H}^{n-1} \text {-almost everywhere on } \partial^{*} E \text {. }
$$

This proposition justifies Definition 1.1. The proof of the proposition relies on the following lemma, which extends arguments from [17] and [18]. Let $\mu$ and $\vec{H}_{\mu}$ be as in Proposition 3.1. On any subset of the support of $\mu$ that is the graph of a measurable function, the mean curvature of $\mu$ is already determined by the mean curvature of the graph function.

LEMMA 3.2 Let $\mu$ be an integral ( $n-1)$-varifold with locally bounded first variation and mean curvature vector $\vec{H}_{\mu} \in \mathrm{L}_{\text {loc }}^{s}(\mu), s>n-1, s \geqslant 2$. If there is a measurable map $\psi: Y \rightarrow \mathbb{R}$, $Y \subset \mathbb{R}^{n-1}$, with

$$
\Psi(y):=(y, \psi(y)) \in \operatorname{spt}(\mu)
$$

for all $y \in Y$ then at $\mathcal{L}^{n-1}$-almost all $y \in Y, \psi$ is twice approximately differentiable and

$$
\vec{H}_{\mu}(\Psi(y))=\nabla \cdot\left(\frac{\nabla \psi}{\sqrt{1+|\nabla \psi|^{2}}}\right)(y) \frac{(-\nabla \psi(y), 1)}{\sqrt{1+|\nabla \psi(y)|^{2}}} .
$$

Proof. Let $\Sigma=\operatorname{spt}(\mu)$ and $\omega: \mathbb{R} \rightarrow \mathbb{R}$ indicate a generic modulus of continuity, that is, an $o(1)$ function at zero. Define the set of "good" points to be

$$
G:=\{y \in Y: \psi \text { is twice approximately differentiable at } y \text { and satisfies } 3.1\}\} .
$$

According to [7, 3.1.4] the set $G$ is measurable, and [7, 2.9.11] ensures that

$$
\theta^{n-1}(G, y)=0 \quad \text { for } \mathcal{L}^{n-1} \text {-almost all } y \in Y \backslash G .
$$

We will show that on the other hand

$$
\theta^{n-1}(G, y)=1 \quad \text { for } \mathcal{L}^{n-1} \text {-almost all } y \in Y .
$$

Comparing $\sqrt{3.2}$ and $\sqrt{3.3}$ we obtain $\mathcal{L}^{n-1}(Y \backslash G)=0$ and the conclusion of the lemma follows. The proof of (3.3) splits into several steps. 
STEP 1 For $\mathcal{L}^{n-1}$-almost all points $y_{0} \in Y$ we find that

$$
\begin{gathered}
x_{0}=\Psi\left(y_{0}\right) \text { is generic with respect to } \mu, \\
\lambda:=\left|\nu_{0} \cdot \vec{e}_{n}\right|>0, \quad \text { where } \nu_{0} \perp T_{x_{0}} \mu,\left|\nu_{0}\right|=1, \\
\psi \text { is approximately differentiable at } y_{0} .
\end{gathered}
$$

Proof. Observe that for the orthogonal projection $\pi: \mathbb{R}^{n} \rightarrow \mathbb{R}^{n-1} \times\{0\}$ and an $(n-1)$-dimensional hyperplane $T=\{v(T)\}^{\perp}$ of $\mathbb{R}^{n}$ the Jacobian is given by

$$
J_{T} \pi=\left|v(T) \cdot \vec{e}_{n}\right| \text {. }
$$

Thus the coarea formula [6, Theorem 3.4.2] guarantees that

$$
\mathcal{L}^{n-1}\left(\left\{y \in Y: v\left(T_{\Psi(y)} \mu\right) \cdot \vec{e}_{n}=0\right\}\right)=0
$$

and

$$
\begin{aligned}
& \mathcal{L}^{n-1}(\{y \in Y: \Psi(y) \text { is not generic with respect to } \mu\}) \\
& \qquad \leqslant \mu(\{x \in \Sigma: x \text { is not generic with respect to } \mu\})=0,
\end{aligned}
$$

according to the rectifiability of $\mu$ and [7, 2.9.11]. Moreover $\psi$ is approximately continuous at $\mathcal{L}^{n-1}$ almost all points $y \in Y$ since $\psi$ is measurable (see [7, 2.9.13]).

STEP 2 Fix $y_{0} \in Y$ with (3.4)-(3.6), set $x_{0}=\Psi\left(y_{0}\right)$. There is $\varrho_{0}=\varrho_{0}(\lambda)>0$ such that equation 3.1) holds at $\mathcal{L}^{n-1}$-almost all points $y \in B_{Q_{0}}\left(y_{0}\right)$ for which $\psi$ coincides with the upper height function of $\mu$ (see the definition below).

Proof. Abbreviate the height of $x_{0}$, the tangential plane of $\mu$ at $x_{0}$ and the $(n-1)$-dimensional density of $\mu$ at $x_{0}$ by

$$
t_{0}:=\psi\left(y_{0}\right), \quad T_{0}:=T_{x_{0}} \mu, \quad \theta_{0}:=\theta^{n-1}\left(\mu, x_{0}\right) .
$$

Denote the "slope" of $T_{0}$ with respect to $\mathbb{R}^{n-1} \times\{0\}$ by

$$
m:=m(\lambda)=\frac{\sqrt{1-\lambda^{2}}}{\lambda} .
$$

By [19, Lemma 17.11] the existence of $T_{x_{0}} \mu$ and $\vec{H}_{\mu} \in \mathrm{L}_{\text {loc }}^{s}(\mu)$ with $s>n-1$ implies

$$
\lim _{\varrho \rightarrow 0}\left(\sup \left\{\frac{1}{\varrho} \operatorname{dist}\left(x, x_{0}+T_{x_{0}} \mu\right): x \in B_{\varrho}^{n}\left(x_{0}\right) \cap \Sigma\right\}\right)=0 .
$$

Therefore we can choose $\varrho_{0}=\varrho_{0}(\lambda)>0$ such that $Z_{\varrho_{0}, 3 m \varrho_{0}}\left(x_{0}\right) \subset \subset \Omega$ and for all $0<\varrho<\varrho_{0}$,

$$
\Sigma \cap Z_{\varrho, 3 m \varrho_{0}}\left(x_{0}\right) \subset Z_{\varrho, 2 m \varrho}\left(x_{0}\right) .
$$

We define the upper and lower height functions

$$
\varphi_{+}: B_{\varrho_{0}}^{n-1}\left(y_{0}\right) \rightarrow[-\infty, \infty), \quad \varphi_{-}: B_{\varrho_{0}}^{n-1}\left(y_{0}\right) \rightarrow(-\infty, \infty],
$$


by

$$
\begin{aligned}
& \varphi_{+}(y):=\sup \left\{t \in\left(t_{0}-3 m \varrho_{0}, t_{0}+3 m \varrho_{0}\right):(y, t) \in \Sigma\right\} \\
& \varphi_{-}(y):=\inf \left\{t \in\left(t_{0}-3 m \varrho_{0}, t_{0}+3 m \varrho_{0}\right):(y, t) \in \Sigma\right\} .
\end{aligned}
$$

Then $\varphi_{+}$is upper and $\varphi_{-}$is lower semicontinuous. By [18, Theorem 6.1] the height function $\varphi_{+}$is twice approximately differentiable $\mathcal{L}^{n-1}$-almost everywhere in $B_{\varrho_{0}}^{n-1}\left(y_{0}\right)$, and for $\mathcal{L}^{n-1}$-almost all $y \in B_{\varrho_{0}}^{n-1}\left(y_{0}\right) \cap\left\{\varphi_{+} \in \mathbb{R}\right\}$,

$$
\vec{H}_{\mu}\left(\left(y, \varphi_{+}(y)\right)\right)=\left(\nabla \cdot \frac{\nabla \varphi_{+}}{\sqrt{1+\left|\nabla \varphi_{+}\right|^{2}}}\right)(y) \frac{\left(-\nabla \varphi_{+}(y), 1\right)}{\sqrt{1+\left|\nabla \varphi_{+}(y)\right|^{2}}} .
$$

Now $\varphi_{+}$and $\psi$ are measurable, and by [7, 2.9.11], $\mathcal{L}^{n-1}$-almost all points in $\left\{\varphi_{+}=\psi\right\}$ have full density in this set. Thus $\psi$ is twice approximately differentiable $\mathcal{L}^{n-1}$-almost everywhere in $\left\{\varphi_{+}=\psi\right\} \cap B_{\varrho_{0}}\left(y_{0}\right)$ and satisfies 3.1 .

STEP 3 The set

$$
\Sigma_{0}:=\left\{\left(y, \varphi_{ \pm}(y)\right): y \in B_{\varrho 0}^{n-1}\left(y_{0}\right), \varphi_{+}(y)=\varphi_{-}(y)\right\}
$$

has full density at $x_{0}$ with respect to $\mu$.

Proof. We follow [17] and use a version of the Lipschitz approximation theorem of Brakke (see A.1 in the appendix). The scaled measures $\mu_{x_{0}, \varrho}$ as defined in 2.1) are integral $(n-1)$-varifolds in $B_{7}^{n}(0)$ with $\mu_{x_{0}, \varrho}\left(B_{7}^{n}(0)\right) \leqslant 2 \omega_{n-1} 7^{n-1} \theta_{0}$ for all $0<\varrho \ll 1$. We have to control the Lipschitz approximation constant (see the definition in the appendix). We claim

$$
\operatorname{lipapp}_{\mu_{x_{0}, \varrho}}\left(0,7, T_{0}\right) \rightarrow 0 \quad(\varrho \rightarrow 0) .
$$

In fact the existence of $T_{0}=T_{x_{0}} \mu$ yields

$$
\int_{B_{7}^{n}(0)} \operatorname{dist}\left(x, T_{0}\right)^{2} \mathrm{~d} \mu_{x_{0}, \varrho} \rightarrow \int_{B_{7}^{n}(0)} \operatorname{dist}\left(x, T_{0}\right)^{2} \mathrm{~d}\left(\theta_{0} \mathcal{H}^{n-1}\left\llcorner T_{0}\right)(x)=0 .\right.
$$

Next we observe that lipapp ${ }_{\mu_{x_{0}, \varrho}}\left(0,7, T_{0}\right)=\operatorname{lipapp}_{\mu}\left(x_{0}, 7 \varrho, T_{0}\right)$ and

$$
(7 \varrho)^{-n+1} \int_{B_{7_{\varrho}}^{n}\left(x_{0}\right)}\left\|T_{x} \mu-T_{0}\right\|^{2} \mathrm{~d} \mu(x) \rightarrow \theta_{0} \omega_{n-1}\left\|T_{x_{0}} \mu-T_{0}\right\|^{2}=0 .
$$

The third term of lipapp $\mu_{x_{0}, \varrho}\left(0,7, T_{0}\right)$ is estimated by

$$
\begin{aligned}
(7 \varrho)^{-n+3} \int_{B_{7 \varrho}^{n}\left(x_{0}\right)}\left|\vec{H}_{\mu}\right|^{2} \mathrm{~d} \mu & \leqslant(7 \varrho)^{-n+3}\left(\int_{B_{7 \varrho}^{n}\left(x_{0}\right)}\left|\vec{H}_{\mu}\right|^{s} \mathrm{~d} \mu\right)^{2 / s} \mu\left(B_{7 \varrho}^{n}\left(x_{0}\right)\right)^{1-2 / s} \\
& \leqslant C\left((7 \varrho)^{-n+1} \mu\left(B_{7 \varrho}^{n}\left(x_{0}\right)\right)\right)^{1-2 / s}(7 \varrho)^{2(1-(n-1) / s)} \rightarrow 0 .
\end{aligned}
$$

Thus the assumptions of Theorem A.1 are fulfilled with $\theta_{0}=\theta^{n-1}\left(\mu, x_{0}\right), T_{0}=T_{x_{0}} \mu$, and 3.8 . Hence there are a constant $\delta_{0}$ independent of $\varrho$ and $\theta_{0}$, and Lipschitz-continuous functions

$$
f_{i}^{\varrho}: B_{\delta_{0}}^{n-1}(0) \rightarrow \mathbb{R}, \quad i=1, \ldots, \theta_{0},
$$


such that for the set $Y_{0}^{\varrho}$ of all $y \in B_{\delta_{0}}^{n-1}(0)$ with

$$
\theta^{n-1}\left(\mu_{x_{0}, \varrho},(y, t)\right)=\#\left\{i: f_{i}^{\varrho}(y)=t\right\} \quad \text { for all }-1 / 2<t<1 / 2
$$

and the set

$$
X_{0}^{\varrho}:=\operatorname{spt}\left(\mu_{x_{0}, \varrho}\right) \cap\left(Y_{0}^{\varrho} \times(-1 / 2,1 / 2)\right)
$$

the following limit vanishes:

$$
\mu_{x_{0}, \varrho}\left(Z_{\delta_{0}, 1 / 2}(0) \backslash X_{0}^{\varrho}\right)+\mathcal{L}^{n-1}\left(B_{\delta_{0}}^{n-1}(0) \backslash Y_{0}^{\varrho}\right) \rightarrow 0 \quad(\varrho \rightarrow 0) .
$$

Assuming $0<\delta_{0}<1 / 4 m$, we observe that, for all $0<\varrho \ll 1$,

$$
\begin{aligned}
Z_{\varrho \delta_{0}, 2 m \varrho \delta_{0}}\left(x_{0}\right) & \subset Z_{\varrho \delta_{0}, \varrho / 2}\left(x_{0}\right), \\
Z_{\varrho \delta_{0}, \varrho / 2}\left(x_{0}\right) & \subset Z_{\varrho 0,3 m \varrho 0}\left(x_{0}\right) .
\end{aligned}
$$

For $x \in\left(x_{0}+\varrho X_{0}^{\varrho}\right) \cap\left\{\theta^{n-1}(\mu, \cdot)=\theta_{0}\right\}, x=(y, t)$, we see from 3.9 that

$$
\begin{gathered}
t=t_{0}+\varrho f_{1}^{\varrho}\left(\frac{y-y_{0}}{\varrho}\right)=\cdots=t_{0}+\varrho f_{\theta_{0}}^{\varrho}\left(\frac{y-y_{0}}{\varrho}\right), \\
\{x\}=\Sigma \cap\left(\{y\} \times\left(t_{0}-\varrho / 2, t_{0}+\varrho / 2\right)\right) .
\end{gathered}
$$

By (3.12) we obtain $x \in Z_{\varrho_{0}, 3 m \varrho_{0}}\left(x_{0}\right)$ and $\varphi_{+}(y), \varphi_{-}(y) \in \mathbb{R}$. Moreover 3.7$)$ and 3.11 yield

$$
\varphi_{+}(y), \varphi_{-}(y) \in\{y\} \times\left(t_{0}-\varrho / 2, t_{0}+\varrho / 2\right)
$$

and by $(3.13)$ we get $\varphi_{-}(y)=\varphi_{+}(y)$ and $x \in \Sigma_{0}$.

Now for $\alpha=\min \left(\delta_{0}, 1 / 2\right)$ and all $0<\varrho<\varrho_{0}$ we have

$$
\begin{aligned}
\mu\left(B_{\alpha \varrho}^{n}\left(x_{0}\right) \backslash \Sigma_{0}\right) & \leqslant \mu\left(B_{\alpha \varrho}^{n}\left(x_{0}\right) \backslash\left(\left(x_{0}+\varrho X_{0}^{\varrho}\right) \cap\left\{\theta^{n-1}(\mu, \cdot)=\theta_{0}\right\}\right)\right) \\
& \leqslant \mu\left(B_{\alpha \varrho}^{n}\left(x_{0}\right) \backslash\left(x_{0}+\varrho X_{0}^{\varrho}\right)\right)+\mu\left(B_{\alpha \varrho}^{n}\left(x_{0}\right) \backslash\left\{\theta^{n-1}(\mu, \cdot)=\theta_{0}\right\}\right) \\
& \leqslant \varrho^{n-1} \mu_{x_{0}, \varrho}\left(Z_{\delta_{0}, 1 / 2}(0) \backslash X_{0}^{\varrho}\right)+\mu\left(B_{\alpha \varrho}^{n}\left(x_{0}\right) \backslash\left\{\theta^{n-1}(\mu, \cdot)=\theta_{0}\right\}\right) \\
& =\varrho^{n-1} \omega(\varrho)+\theta_{0} \omega_{n-1}(\alpha \varrho)^{n-1} \omega(\varrho),
\end{aligned}
$$

where we have used 3.10 , the full density with respect to $\mu$ of the set $\left\{\theta^{n-1}(\mu, \cdot)=\theta_{0}\right\}$ at $x_{0}$ according to assumption (3.4), and $\theta_{0}=\theta^{n-1}\left(\mu, x_{0}\right)$. The above calculations yield the claim of Step 3,

$$
\mu\left(B_{\alpha \varrho}^{n}\left(x_{0}\right) \backslash \Sigma_{0}\right) \leqslant \varrho^{n-1} \omega(\varrho) .
$$

STEP 4 The set $\left\{\varphi_{+}=\psi\right\}$ has full density at $y_{0}$.

Proof. For $(y, \psi(y)) \in Z_{\varrho_{0}, 3 m \varrho_{0}}\left(x_{0}\right)$ we have

$$
\varphi_{-}(y) \leqslant \psi(y) \leqslant \varphi_{+}(y) .
$$


Thus

$$
\begin{aligned}
\mathcal{L}^{n-1}\left(\left\{\varphi_{+} \neq \psi\right\}\right. & \left.\cap B_{\varrho}^{n-1}\left(y_{0}\right)\right) \\
& \leqslant \mathcal{L}^{n-1}\left(\left\{\Psi \notin Z_{\varrho, 3 m \varrho_{0}}\left(x_{0}\right)\right\}\right)+\mathcal{L}^{n-1}\left(\left\{\Psi \in Z_{\varrho, 3 m \varrho_{0}}\left(x_{0}\right)\right\} \cap\left\{\varphi_{+} \neq \varphi_{-}\right\}\right)
\end{aligned}
$$

The approximate continuity of $\psi$ at $y_{0}$ implies

$$
\mathcal{L}^{n-1}\left(\left\{\Psi \notin Z_{\varrho, 3 m \varrho_{0}}\left(x_{0}\right)\right\}\right)=\varrho^{n-1} \omega(\varrho) .
$$

The coarea formula and 3.7 yield, with $\beta=2 \max \{2 m, 1\}$,

$$
\begin{aligned}
\mathcal{L}^{n-1}\left(\left\{\Psi \in Z_{\varrho, 3 m \varrho_{0}}\left(x_{0}\right)\right\} \cap\left\{\varphi_{+} \neq \varphi_{-}\right\}\right) \leqslant & \mathcal{L}^{n-1}\left(\pi\left(\left(\Sigma \cap Z_{\varrho, 2 m \varrho}\left(x_{0}\right)\right) \backslash \Sigma_{0}\right)\right) \\
\leqslant & \int_{\left(\Sigma \cap Z_{\varrho, 2 m \varrho}\left(x_{0}\right)\right) \backslash \Sigma_{0}}\left|v\left(T_{x} \mu\right) \cdot \vec{e}_{n}\right| \mathrm{d} \mathcal{H}^{n-1}(x) \\
\leqslant & \int_{B_{\beta \varrho}^{n}\left(x_{0}\right) \backslash \Sigma_{0}} 1 \mathrm{~d} \mu=\varrho^{n-1} \omega(\varrho),
\end{aligned}
$$

where we have used (3.14). Together with 3.16 and 3.17 we end up with

$$
\theta^{n-1}\left(\left\{\varphi_{+} \neq \psi\right\}, y_{0}\right)=0
$$

STEP 5 Steps 4 and 2 prove that $\psi$ is twice approximately differentiable in a set of full $\mathcal{L}^{n-1}$ density at $y_{0}$ and satisfies 3.1 . Thus we arrive at 3.3 , which completes the proof of the lemma.

Now we are in a position to prove Proposition 3.1

Proof of Proposition 3.1 Since $\mathcal{X}_{E} \in \mathrm{BV}(\Omega)$ and the reduced boundary of $E$ is $(n-1)$-rectifiable, $\partial^{*} E$ is up to a set of $\mathcal{H}^{n-1}$-measure zero covered by a countable union of Lipschitz $(n-1)$ graphs (see [3, Proposition 2.76]). Lemma 3.2 ensures that for each of these Lipschitz graphs the mean curvature vectors $\vec{H}_{\mu_{1}}$ and $\vec{H}_{\mu_{2}}$ on the intersection of the graph with $\partial^{*} E$ are $\mathcal{H}^{n-1}$-almost everywhere determined by the graph function and therefore identical. Thus, as desired,

$$
\vec{H}_{\mu_{1}}=\vec{H}_{\mu_{2}} \quad \mathcal{H}^{n-1} \text {-almost everywhere on } \partial^{*} E .
$$

A generalised solution $(u, \mathcal{X})$ of problem $(1.1),(1.2)$ should fulfil the Gibbs-Thomson law in the sense that for almost all $t \in(0, T)$ the phase interface $\partial^{*}\{\mathcal{X}(t, \cdot)=1\}$ has a mean curvature vector according to Definition 1.1 which is $\mathcal{H}^{n-1}$-almost everywhere on $\partial^{*}\{\mathcal{X}(t, \cdot)=1\}$ given by the trace of $u$ as $\vec{H}(t, \cdot)=u(t, \cdot) \frac{\nabla \mathcal{X}}{|\nabla \mathcal{X}|}(t, \cdot)$.

In fact this is a reasonable formulation of the Gibbs-Thomson law.

Proposition 3.3 Let $u \in \mathrm{H}^{1,2}(\Omega)$ and $\mathcal{X} \in \operatorname{BV}(\Omega)$,

(i) For $\mathcal{H}^{n-1}$-almost all $x \in \Omega$ the mean values

$$
\lim _{r \rightarrow 0} \frac{1}{\left|B_{r}(x)\right|} \int_{B_{r}(x)} u(y) \mathrm{d} y
$$

of $u$ exists. In this sense for all $(n-1)$-rectifiable sets a trace of $u$ is given. 
(ii) If $n=2,3$ and $(u, \mathcal{X})$ satisfies the Gibbs-Thomson law in the BV-formulation, then the phase interface $\partial^{*}\{\mathcal{X}=1\}$ has a mean curvature vector according to Definition 1.1 and the equation $\vec{H}=u \nabla \mathcal{X} /|\nabla \mathcal{X}|$ holds $\mathcal{H}^{n-1}$-almost everywhere on $\partial^{*}\{\mathcal{X}=1\}$.

(iii) If $n=2,3$ and $\mu=|\nabla \mathcal{X}|$ is an integral varifold with locally bounded first variation and mean curvature $\vec{H}_{\mu} \in \mathrm{L}_{\text {loc }}^{s}(\mu), s>n-1$, satisfying $\vec{H}_{\mu}=u \nabla \mathcal{X} /|\nabla \mathcal{X}| \mu$-almost everywhere, then $(u, \mathcal{X})$ is a solution of the Gibbs-Thomson law in the BV-formulation.

Proof. (i) The existence of the mean value limits on a set of vanishing 2-capacity and in particular $\mathcal{H}^{n-1}$-almost everywhere follows from [6, 4.7.2 and 4.8].

(ii) For $\xi \in C_{\mathrm{c}}^{\infty}(\Omega)$ and $\mu=|\nabla \mathcal{X}|$ we have

$$
\delta \mu(\xi)=\int_{\Omega} \operatorname{div}_{T_{x} \mu} \xi(x) \mathrm{d} \mu(x)=\int_{\Omega}\left(\nabla \cdot \xi-\frac{\nabla \mathcal{X}}{|\nabla \mathcal{X}|} \cdot D \xi \frac{\nabla \mathcal{X}}{|\nabla \mathcal{X}|}\right)|\nabla \mathcal{X}|
$$

Invoking [17, Theorem 1.3] we find that $\mu$ has locally bounded first variation with mean curvature vector $\vec{H}_{\mu} \in \mathrm{L}_{\text {loc }}^{s}(\mu), s>n-1$. We obtain $u \in \mathrm{L}_{\text {loc }}^{s}(\mu)$ and

$$
-\int_{\Omega} \vec{H}_{\mu} \cdot \xi \mathrm{d} \mu=\int_{\Omega} \nabla \cdot(u \xi) \mathcal{X}=-\int_{\Omega} u \frac{\nabla \mathcal{X}}{|\nabla \mathcal{X}|} \cdot \xi \mathrm{d} \mu .
$$

Thus $\vec{H}_{\mu}=u \nabla \mathcal{X} /|\nabla \mathcal{X}| \mu$-almost everywhere. Definition 1.1 and $\mathcal{H}^{n-1}\left\llcorner\partial^{*}\{\mathcal{X}=1\} \leqslant \mu\right.$ prove the claim.

(iii) From 3.18 we deduce that

$$
\int_{\Omega}\left(\nabla \cdot \xi-\frac{\nabla \mathcal{X}}{|\nabla \mathcal{X}|} \cdot \frac{\nabla \mathcal{X}}{|\nabla \mathcal{X}|}\right)|\nabla \mathcal{X}|=\int_{\Omega}-u \frac{\nabla \mathcal{X}}{|\nabla \mathcal{X}|} \cdot \xi \mathrm{d} \mu=\int_{\Omega} \nabla \cdot(u \xi) \mathcal{X}
$$

which is the desired BV-formulation.

\section{Time-discretisation and local minimisation}

We determine approximate solutions by an implicit time-discretisation. Compared to [11] we take the same time-discrete functionals, but choose phase functions to be suitable stationary points and not necessarily global minimisers.

Let a time step $h>0$ be given and look for step functions in time

$$
u^{h}:(0, T) \rightarrow\left(u_{\mathrm{D}}+M_{0}\right) \cap \mathrm{L}^{\infty}(\Omega), \quad \mathcal{X}^{h}:(0, T) \rightarrow \mathrm{BV}(\Omega ;\{0,1\}) .
$$

We write $u_{t}^{h}=u^{h}(t, \cdot), \mathcal{X}_{t}^{h}=\mathcal{X}^{h}(t, \cdot)$ and denote the backward difference quotient of a time dependent function $w$ by $\partial_{t}^{-h} w(t):=\frac{1}{h}(w(t)-w(t-h))$. We prescribe the implicit time-discrete energy balance equation

$$
\partial_{t}^{-h}\left(u^{h}+\mathcal{X}^{h}\right)(t)-\Delta u_{t}^{h}=f,\left.\quad u_{t}^{h}\right|_{\Gamma_{\mathrm{D}}}=u_{\mathrm{D}}
$$

This turns out to be equivalent to

$$
u_{t}^{h}=K^{h}\left(u_{t-h}^{h}\right)-K_{0}^{h}\left(\mathcal{X}_{t}^{h}-\mathcal{X}_{t-h}^{h}\right)+h K_{0}^{h}(f)
$$


if for $v \in \mathrm{L}^{1}(\Omega)$ the functions $K_{0}^{h}(v), K^{h}(v)$ are defined as solutions of

$$
\begin{aligned}
& K_{0}^{h}(v)-h \Delta K_{0}^{h}(v)=v,\left.\quad K_{0}^{h}(v)\right|_{\Gamma_{\mathrm{D}}}=0,\left.\quad \nabla K_{0}^{h}(v) \cdot v_{\Omega}\right|_{\partial \Omega \backslash \Gamma_{\mathrm{D}}}=0, \\
& K^{h}(v)-h \Delta K^{h}(v)=v,\left.\quad K^{h}(v)\right|_{\Gamma_{\mathrm{D}}}=u_{\mathrm{D}},\left.\quad \nabla K^{h}(v) \cdot v_{\Omega}\right|_{\partial \Omega \backslash \Gamma_{\mathrm{D}}}=0 .
\end{aligned}
$$

For future use we also define $\tilde{K}^{h}(v)$ to be the solution of

$$
\tilde{K}^{h}(v)-h \Delta \tilde{K}^{h}(v)=v,\left.\quad \nabla \tilde{K}^{h}(v)\right|_{\partial \Omega} \cdot v_{\Omega}=0 .
$$

Let us first summarise some properties of the above operators.

LEMmA 4.1 The maps $K^{h}, K_{0}^{h}, \tilde{K}^{h}: \mathrm{L}^{2} \rightarrow \mathrm{H}^{1,2}(\Omega)$ are continuous. For $v \in \mathrm{L}^{\infty}(\Omega)$ we have

$$
\begin{aligned}
& \left\|K^{h}(v)\right\|_{\mathrm{L}^{\infty}(\Omega)} \leqslant \max \left(\|v\|_{\mathrm{L}^{\infty}(\Omega)}+\left\|u_{\mathrm{D}}\right\|_{\mathrm{L}^{\infty}(\partial \Omega)}\right), \\
& \left\|K_{0}^{h}(v)\right\|_{\mathrm{L}^{\infty}(\Omega)},\left\|\tilde{K}^{h}(v)\right\|_{\mathrm{L}^{\infty}(\Omega)} \leqslant\|v\|_{\mathrm{L}^{\infty}(\Omega)} .
\end{aligned}
$$

The operators $(v, w) \mapsto \frac{1}{2} \int_{\Omega} K_{0}^{h}(v) w$ and $(v, w) \mapsto \frac{1}{2} \int_{\Omega} K_{0}^{h}(v) w$ acting on $\mathrm{L}^{\infty}(\Omega) \times \mathrm{L}^{\infty}(\Omega)$ are bilinear, symmetric and continuous in each variable with respect to $\mathrm{L}^{1}(\Omega)$-convergence; the corresponding quadratic form is uniformly bounded in $h>0$ on bounded sets in $\mathrm{L}^{\infty}(\Omega)$ and continuous with respect to $\mathrm{L}^{1}(\Omega)$ convergence.

Proof. To prove $K^{h}(v) \geqslant-M:=\min \left(\inf _{\Omega} v, \inf _{\partial \Omega} u_{\mathrm{D}}\right)$ test 4.3 with $\left(-K^{h}(v)-M\right)_{+}$. Using $v, u_{\mathrm{D}} \geqslant-M$ we get

$$
\begin{aligned}
0= & \int_{\Omega}\left(K^{h}(v)-v\right)\left(-K^{h}(v)-M\right)_{+}+h \nabla K^{h}(v) \cdot \nabla\left(-K^{h}(v)-M\right)_{+} \\
& -\int_{\partial \Omega} h\left(-K^{h}(v)-M\right)_{+} \nabla K^{h}(v) \cdot v_{\Omega} \\
\leqslant & -\int_{\Omega}\left(-K^{h}(v)-M\right)_{+}^{2}+h\left|\nabla\left(-K^{h}(v)-M\right)_{+}\right|^{2}
\end{aligned}
$$

and we obtain $K^{h}(v) \geqslant-M$ almost everywhere in $\Omega$. The corresponding estimate from above and $\mathrm{L}^{\infty}(\Omega)$-bounds for $K_{0}^{h}(v)$ and $\tilde{K}^{h}(v)$ can be derived analogously. The proofs of the other assertions are straightforward.

We determine the functions $u^{h}, \mathcal{X}^{h}$ iteratively. Set

$$
u_{t}^{h}=u_{0}, \quad \mathcal{X}_{t}^{h}=\mathcal{X}_{0} \quad \text { for } 0 \leqslant t<h
$$

and define, for known $u_{t-h}^{h}, \mathcal{X}_{t-h}^{h}$ functionals $F_{t}^{h}: \operatorname{BV}(\Omega ;\{0,1\}) \rightarrow \mathbb{R}$,

$$
F_{t}^{h}(\mathcal{X})=\int_{\Omega}|\nabla \mathcal{X}|-\int_{\Omega}\left(K^{h}\left(u_{t-h}^{h}\right) \mathcal{X}-\frac{1}{2} K_{0}^{h}\left(\mathcal{X}-\mathcal{X}_{t-h}^{h}\right)\left(\mathcal{X}-\mathcal{X}_{t-h}^{h}\right)\right)
$$

In [11] the function $\mathcal{X}_{t}^{h}$ is chosen to be a global minimiser of $F_{t}^{h}$, and $u_{t}^{h}$ is defined by 4.1 . Let us make a few remarks concerning this approach:

- The first variation of the area integral $\int_{\Omega}|\nabla \mathcal{X}|$ is given by the mean curvature, leading to an approximate Gibbs-Thomson law for stationary points of $F_{t}^{h}$. 
- If for $h \leqslant t \leqslant t_{0}$ the $\mathcal{X}_{t}^{h}$ are stationary points of the functionals $F_{t}^{h}$ and in addition $F_{t}^{h}\left(\mathcal{X}_{t}^{h}\right) \leqslant$ $F_{t}^{h}\left(\mathcal{X}_{t-h}^{h}\right)$, then we get an energy estimate

$$
\int_{\Omega}\left|\nabla \mathcal{X}_{t_{0}}^{h}\right|+\frac{1}{2} \int_{\Omega}\left(u_{t_{0}}^{h}\right)^{2}+\frac{1}{2} \int_{0}^{t_{0}} \int_{\Omega}\left|\nabla u_{t}^{h}\right|^{2} \mathrm{~d} t \leqslant C
$$

with a constant $C$ independent of $t_{0}, h$ (see [11]).

- The term $\frac{1}{2} \int_{\Omega} K_{0}^{h}\left(\mathcal{X}-\mathcal{X}_{t-h}^{h}\right)\left(\mathcal{X}-\mathcal{X}_{t-h}^{h}\right)$ "penalises" in some sense the distance from $\mathcal{X}_{t-h}^{h}$. As we see from Lemma 4.1 the penalisation is uniformly bounded in $h>0$ and consequently quite mild. Thus global minimisation preserves a minimising property for the limits of the approximate solutions. This excludes a loss of surface area of phase interfaces as $h \rightarrow 0$ and gives additional regularity properties.

If we consider the functional $F_{t}^{h}$ as a kind of energy of the physical system, it is not very well justified to choose $\mathcal{X}_{t}^{h}$ to be a global minimiser. A global minimiser of $F_{t}^{h}$ can be "far away" from $\mathcal{X}_{t-h}^{h}$, with a large amount of energy "in between". It would be more appropriate to choose a local minimum connected with $\mathcal{X}_{t-h}^{h}$ by a path on which the energy monotonically decreases.

To implement this idea we approximate a flow $\tilde{\mathcal{X}}(\tau), \tau \geqslant 0$, satisfying

$$
\begin{gathered}
\tilde{\mathcal{X}}(0)=\mathcal{X}_{t-h}^{h}, \\
F_{t}^{h}(\tilde{\mathcal{X}}(\tau)) \text { decreasing in } \tau, \\
\tilde{\mathcal{X}}(\tau) \rightarrow \tilde{\mathcal{X}}_{\infty}, \text { where } \tilde{\mathcal{X}}_{\infty} \text { is a stationary point of } F_{t}^{h},
\end{gathered}
$$

by a second discrete evolution $\left(\tilde{\mathcal{X}}_{i}\right)_{i \in \mathbb{N}}$. This can be seen as an evolution on a finer scale. We call this approach local minimisation in contrast to the global minimisation used in [11].

Definition 4.2 Choose positive "time steps" $\left(\varepsilon_{h}\right)_{h>0}$ and "penalisation parameters" $\left(\lambda_{h}\right)_{h>0}$ with

$$
\varepsilon_{h} \rightarrow 0, \quad \lambda_{h} \rightarrow \infty \quad(h \rightarrow 0) .
$$

To determine iteratively $\tilde{\mathcal{X}}_{i}=\tilde{\mathcal{X}}_{t, i}^{h} \in \mathrm{BV}(\Omega,\{0,1\})$ set

$$
\tilde{\mathcal{X}}_{0}:=\mathcal{X}_{t-h}^{h}
$$

and let $\tilde{\mathcal{X}}_{i}$ be a global minimiser of the functional $F_{i}=F_{t, i}^{h}$,

$$
F_{i}(\mathcal{X}):=F_{t}^{h}(\mathcal{X})+\lambda_{h} \int_{\Omega} \tilde{K}^{\varepsilon h}\left(\mathcal{X}-\tilde{\mathcal{X}}_{i-1}\right)\left(\mathcal{X}-\tilde{\mathcal{X}}_{i-1}\right),
$$

where the operator $\tilde{K}^{\varepsilon_{h}}$ was defined in 4.4 .

LEMma 4.3 For $\tilde{\mathcal{X}}_{i-1} \in \operatorname{BV}(\Omega ;\{0,1\})$ there exists a global minimiser $\tilde{\mathcal{X}}_{i}$ of $F_{i}$. After changing $\tilde{\mathcal{X}}_{i}$ on a set of $\mathcal{L}^{3}$-measure zero, $\partial\left\{\tilde{\mathcal{X}}_{i}=1\right\}$ is a $C^{1,1 / 2}$-hypersurface.

Proof. Let $\psi_{j} \in \operatorname{BV}(\Omega ;\{0,1\})$ be a minimising sequence in $\operatorname{BV}(\Omega ;\{0,1\})$. Thus $\left(\psi_{j}\right)_{j \in \mathbb{N}}$ is uniformly bounded in $\operatorname{BV}(\Omega)$. The compact embedding $\mathrm{BV}(\Omega) \hookrightarrow \mathrm{L}^{1}(\Omega)$ implies the strong $\mathrm{L}^{1}(\Omega)$-convergence to a function $\mathcal{X} \in \mathrm{BV}(\Omega ;\{0,1\})$ for a subsequence $j_{k} \rightarrow \infty$. With respect 
to strong $\mathrm{L}^{1}(\Omega)$-convergence the perimeter is lower semicontinuous and all other terms of $\tilde{F}_{i}$ are continuous. This shows that $\mathcal{X}$ is a global minimiser. To prove the regularity of the boundary we show that a global minimiser $\tilde{\mathcal{X}}_{i}$ has a 1/2-almost-minimal boundary in the sense of Almgren.

For this let $\overline{B_{r}(x)} \subset \Omega, \psi \in \operatorname{BV}(\Omega ;\{0,1\})$ with $\tilde{\mathcal{X}}_{i}=\psi$ in $\Omega \backslash \overline{B_{r}(x)}$. The inequality $\tilde{F}_{i}\left(\tilde{\mathcal{X}}_{i}\right) \leqslant \tilde{F}_{i}(\psi)$ leads to the estimate

$$
\begin{aligned}
& \int_{\Omega}\left|\nabla \tilde{\mathcal{X}}_{i}\right|-\int_{\Omega}|\nabla \psi| \\
& \quad \leqslant \int_{\Omega}\left(-K^{h}\left(u_{t-h}^{h}\right)+\frac{1}{2} K_{0}^{h}\left(\tilde{\mathcal{X}}_{i}+\psi-2 \mathcal{X}_{t-h}^{h}\right)+\lambda_{h} \tilde{K}^{\varepsilon_{h}}\left(\tilde{\mathcal{X}}_{i}+\psi-2 \tilde{\mathcal{X}}_{i-1}\right)\right)\left(\tilde{\mathcal{X}}_{i}-\psi\right),
\end{aligned}
$$

where we have used the bilinearity and symmetry of the maps $(v, w) \mapsto \int_{\Omega} K_{0}^{h}(v) w$ and $(v, w) \mapsto$ $\int_{\Omega} K^{\varepsilon h}(v) w$. Lemma 4.1 together with $u_{t-h}^{h} \in \mathrm{L}^{\infty}(\Omega)$ ensures that

$$
\int_{\Omega}\left|\nabla \tilde{\mathcal{X}}_{i}\right|-\int_{\Omega}|\nabla \psi| \leqslant\left(\left\|K^{h}\left(u_{t-h}^{h}\right)\right\|_{L^{\infty}(\Omega)}+1+\lambda_{h}\right) \omega_{3} r^{3} .
$$

According to [22, XI.8] the set $\left\{\tilde{\mathcal{X}}_{i}=1\right\}$ has a $1 / 2$-almost-minimal boundary. By a regularity theorem of Almgren ([2], see [22, XI.8.3]) we conclude that $\partial^{*}\left\{\tilde{\mathcal{X}}_{i}=1\right\}$ is a two-dimensional $C^{1,1 / 2}$-surface with $\partial^{*}\left\{\tilde{\mathcal{X}}_{i}=1\right\}=\partial\left\{\tilde{\mathcal{X}}_{i}=1\right\}$ after changing $\tilde{\mathcal{X}}_{i}$ on a set of measure zero (see $[8,3.1])$.

Next we prove the convergence of $\tilde{\mathcal{X}}_{i}$ up to a subsequence to a stationary point of $F_{t}^{h}$.

Lemma 4.4 There is a subsequence $i_{k} \rightarrow \infty$ and a function $\tilde{\mathcal{X}}_{\infty} \in \mathrm{BV}(\Omega ;\{0,1\})$ such that

$$
\begin{aligned}
\tilde{\mathcal{X}}_{i_{k}} \rightarrow \tilde{\mathcal{X}}_{\infty} & \text { in } \mathrm{L}^{1}(\Omega), \\
\tilde{\mathcal{X}}_{i_{k}-1} \rightarrow \tilde{\mathcal{X}}_{\infty} & \text { in } \mathrm{L}^{1}(\Omega) .
\end{aligned}
$$

Moreover,

$$
F_{t}^{h}\left(\tilde{\mathcal{X}}_{\infty}\right)+\sum_{i=1}^{\infty} \lambda_{h} \int_{\Omega} \tilde{K}^{\varepsilon_{h}}\left(\tilde{\mathcal{X}}_{i}-\tilde{\mathcal{X}}_{i-1}\right)\left(\tilde{\mathcal{X}}_{i}-\tilde{\mathcal{X}}_{i-1}\right) \leqslant F_{t}^{h}\left(\mathcal{X}_{t-h}^{h}\right)
$$

and

$$
F_{t}^{h}\left(\tilde{\mathcal{X}}_{\infty}\right) \leqslant F_{t}^{h}(\mathcal{X})+\lambda_{h} \int_{\Omega} \tilde{K}^{\varepsilon_{h}}\left(\mathcal{X}-\tilde{\mathcal{X}}_{\infty}\right)\left(\mathcal{X}-\tilde{\mathcal{X}}_{\infty}\right) \quad \text { for all } \mathcal{X} \in \mathrm{BV}(\Omega ;\{0,1\})
$$

In particular, $\tilde{\mathcal{X}}_{\infty}$ is a global minimiser of $\tilde{F}: \operatorname{BV}(\Omega ;\{0,1\}) \rightarrow \mathbb{R}$, defined by

$$
\tilde{F}(\mathcal{X}):=F_{t}^{h}(\mathcal{X})+\lambda_{h} \int_{\Omega} \tilde{K}^{\varepsilon_{h}}\left(\mathcal{X}-\tilde{\mathcal{X}}_{\infty}\right)\left(\mathcal{X}-\tilde{\mathcal{X}}_{\infty}\right),
$$

and $\left\{\tilde{\mathcal{X}}_{\infty}=1\right\}$ has a $C^{1,1 / 2}$-boundary after changing $\tilde{\mathcal{X}}_{\infty}$ on a set of $\mathcal{L}^{3}$-measure zero. 
Proof. Observe $F_{j}\left(\tilde{\mathcal{X}}_{j}\right) \leqslant F_{j}\left(\tilde{\mathcal{X}}_{j-1}\right)$ and sum over $j$ to obtain, for any $i \in \mathbb{N}$,

$$
F_{t}^{h}\left(\tilde{\mathcal{X}}_{i}\right)+\sum_{j=1}^{i} \lambda_{h} \int_{\Omega} \tilde{K}^{\varepsilon_{h}}\left(\tilde{\mathcal{X}}_{j}-\tilde{\mathcal{X}}_{j-1}\right)\left(\tilde{\mathcal{X}}_{j}-\tilde{\mathcal{X}}_{j-1}\right) \leqslant F_{t}^{h}\left(\mathcal{X}_{t-h}^{h}\right) .
$$

Thus we can estimate

$$
\begin{aligned}
& \int_{\Omega}\left|\nabla \tilde{\mathcal{X}}_{i}\right|+\sum_{j=1}^{i} \lambda_{h} \int_{\Omega} \tilde{K}^{\varepsilon_{h}}\left(\tilde{\mathcal{X}}_{j}-\tilde{\mathcal{X}}_{j-1}\right)\left(\tilde{\mathcal{X}}_{j}-\tilde{\mathcal{X}}_{j-1}\right) \\
& \quad \leqslant \int_{\Omega}\left|\nabla \mathcal{X}_{t-h}^{h}\right|+\int_{\Omega} K^{h}\left(u_{t-h}^{h}\right)\left(\tilde{\mathcal{X}}_{i}-\mathcal{X}_{t-h}^{h}\right)-\frac{1}{2} \int_{\Omega} K_{0}^{h}\left(\tilde{\mathcal{X}}_{i}-\mathcal{X}_{t-h}^{h}\right)\left(\tilde{\mathcal{X}}_{i}-\mathcal{X}_{t-h}^{h}\right) \\
& \quad \leqslant\left\|\mathcal{X}_{t-h}^{h}\right\|_{\mathrm{BV}(\Omega)}+\left\|K^{h}\left(u_{t-h}^{h}\right)\right\|_{\mathrm{L}^{1}(\Omega)} .
\end{aligned}
$$

Therefore $\left(\tilde{\mathcal{X}}_{i}\right)_{i \in \mathbb{N}}$ is uniformly bounded in $\operatorname{BV}(\Omega)$ and we can choose a subsequence to get $\tilde{\mathcal{X}}_{\infty}, \tilde{\mathcal{X}}_{\infty}^{\prime} \in \mathrm{BV}(\Omega ;\{0,1\})$ with

$$
\begin{aligned}
\tilde{\mathcal{X}}_{i_{k}} \rightarrow \tilde{\mathcal{X}}_{\infty} & \text { in } \mathrm{L}^{1}(\Omega), \\
\tilde{\mathcal{X}}_{i_{k}-1} \rightarrow \tilde{\mathcal{X}}_{\infty}^{\prime} & \text { in } \mathrm{L}^{1}(\Omega) .
\end{aligned}
$$

By 4.10] and Lemma 4.1 we obtain

$$
\begin{aligned}
0 & =\lim _{k \rightarrow \infty} \lambda_{h} \int_{\Omega} \tilde{K}^{\varepsilon_{h}}\left(\tilde{\mathcal{X}}_{i_{k}}-\tilde{\mathcal{X}}_{i_{k}-1}\right)\left(\tilde{\mathcal{X}}_{i_{k}}-\tilde{\mathcal{X}}_{i_{k}-1}\right) \\
& =\lambda_{h} \int_{\Omega} \tilde{K}^{\varepsilon_{h}}\left(\tilde{\mathcal{X}}_{\infty}-\tilde{\mathcal{X}}_{\infty}^{\prime}\right)\left(\tilde{\mathcal{X}}_{\infty}-\tilde{\mathcal{X}}_{\infty}^{\prime}\right) \\
& =\lambda_{h} \int_{\Omega} \tilde{K}^{\varepsilon_{h}}\left(\tilde{\mathcal{X}}_{\infty}-\tilde{\mathcal{X}}_{\infty}^{\prime}\right)^{2}+\varepsilon_{h} \lambda_{h} \int_{\Omega}\left|\nabla \tilde{K}^{\varepsilon_{h}}\left(\tilde{\mathcal{X}}_{\infty}-\tilde{\mathcal{X}}_{\infty}^{\prime}\right)\right|^{2}
\end{aligned}
$$

and consequently $\tilde{\mathcal{X}}_{\infty}=\tilde{\mathcal{X}}_{\infty}^{\prime}$. Now $F_{t}^{h}$ is lower semicontinuous with respect to $\mathrm{L}^{1}$-convergence and by means of (4.9) we get the estimate (4.7).

Recalling $F_{i_{k}}\left(\tilde{\mathcal{X}}_{i_{k}}\right) \leqslant F_{i_{k}}(\mathcal{X})$ for any $\mathcal{X} \in \operatorname{BV}(\Omega ;\{0,1\})$, we have

$$
\begin{aligned}
F_{t}^{h}\left(\tilde{\mathcal{X}}_{i_{k}}\right)+\lambda_{h} \int_{\Omega} \tilde{K}^{\varepsilon_{h}}\left(\tilde{\mathcal{X}}_{i_{k}}-\tilde{\mathcal{X}}_{i_{k}-1}\right)\left(\tilde{\mathcal{X}}_{i_{k}}\right. & \left.-\tilde{\mathcal{X}}_{i_{k}-1}\right) \\
& \leqslant F_{t}^{h}(\mathcal{X})+\lambda_{h} \int_{\Omega} \tilde{K}^{\varepsilon_{h}}\left(\mathcal{X}-\tilde{\mathcal{X}}_{i_{k}-1}\right)\left(\mathcal{X}-\tilde{\mathcal{X}}_{i_{k}-1}\right) .
\end{aligned}
$$

Letting $k \rightarrow \infty$ in this estimate gives [4.8. The regularity of the boundary follows as in Lemma 4.3

Definition 4.5 Set

$$
\mathcal{X}_{t}^{h}=\tilde{\mathcal{X}}_{\infty} \quad \text { for an } \tilde{\mathcal{X}}_{\infty} \text { as in Lemma 4.4 }
$$

and define $u_{t}^{h}$ by 4.1 .

We immediately observe that $u_{t}^{h} \in \mathrm{L}^{\infty}(\Omega)$ since $u_{t-h}^{h} \in \mathrm{L}^{\infty}(\Omega)$, and by Lemma 4.1 . 
LEMMA 4.6 For the approximate solutions we have

$$
\begin{gathered}
F_{t}^{h}\left(\mathcal{X}_{t}^{h}\right) \leqslant F_{t}^{h}\left(\mathcal{X}_{t-h}^{h}\right), \\
u_{t}^{h} \in u_{\mathrm{D}}+M_{0}, \quad \nabla u_{t}^{h} \cdot v_{\Omega}=0 \text { on } \partial \Omega \backslash \Gamma_{\mathrm{D}}, \\
\partial_{t}^{-h} u^{h}(t)-\Delta u_{t}^{h}=f .
\end{gathered}
$$

The function $\mathcal{X}_{t}^{h}$ is a stationary point of $F_{t}^{h}$ and for all $\xi \in C_{\mathrm{c}}^{1}\left(\Omega ; \mathbb{R}^{3}\right)$ we have

$$
\int_{\Omega}\left(\nabla \cdot \xi-\frac{\nabla \mathcal{X}_{t}^{h}}{\left|\nabla \mathcal{X}_{t}^{h}\right|} \cdot D \xi \frac{\nabla \mathcal{X}_{t}^{h}}{\left|\nabla \mathcal{X}_{t}^{h}\right|}\right)\left|\nabla \mathcal{X}_{t}^{h}\right|=\int_{\Omega} \mathcal{X}_{t}^{h} \nabla \cdot\left(\left(u_{t}^{h}-h K_{0}^{h}(f)\right) \xi\right) .
$$

Proof. Inequality (4.11) follows from (4.7). We deduce (4.12) from (4.1), (4.2) and (4.3). Since $\mathcal{X}_{t}^{h}=\tilde{\mathcal{X}}_{\infty}$ is a stationary point of $\tilde{F}$ and the first variation of $\mathcal{X} \mapsto \lambda_{h} \int_{\Omega}^{K^{\varepsilon_{h}}}\left(\mathcal{X}-\tilde{\mathcal{X}}_{\infty}\right)\left(\mathcal{X}-\tilde{\mathcal{X}}_{\infty}\right)$ vanishes at $\mathcal{X}_{t}^{h}$, we obtain

$$
0=\int_{\Omega}\left(\nabla \cdot \xi-\frac{\nabla \mathcal{X}_{t}^{h}}{\left|\nabla \mathcal{X}_{t}^{h}\right|} \cdot D \xi \frac{\nabla \mathcal{X}_{t}^{h}}{\left|\nabla \mathcal{X}_{t}^{h}\right|}\right)\left|\nabla \mathcal{X}_{t}^{h}\right|-\int_{\Omega} \mathcal{X}_{t}^{h} \nabla \cdot\left(\left(K^{h}\left(u_{t-h}^{h}\right)-K_{0}^{h}\left(\mathcal{X}_{t}^{h}-\mathcal{X}_{t-h}^{h}\right)\right) \xi\right) .
$$

Now 4.1) gives the assertion.

The following estimates are proved in [11], where only (4.1) and (4.11) are used (and not the global minimising property of the approximate phase functions!).

Lemma 4.7 For any $t_{0} \in(0, T), t_{0}=M h$, we have the energy estimate

$$
\begin{aligned}
\int_{\Omega}\left|\nabla \mathcal{X}_{t_{0}}^{h}\right|+\frac{1}{2} \int_{\Omega}\left(u_{t_{0}}^{h}\right)^{2}+\sum_{j=1}^{M} \frac{h}{2} \int_{\Omega}\left(\left|\nabla u_{j h}^{h}\right|^{2}\right. & \left.+\left|\nabla K^{h}\left(u_{(j-1) h}^{h}\right)\right|^{2}\right) \\
& \leqslant C\left(u_{\mathrm{D}}, f, \Omega\right)+\int_{\Omega}\left|\nabla \mathcal{X}_{0}\right|+\frac{1}{2} \int_{\Omega} u_{0}^{2} .
\end{aligned}
$$

Thus we get uniform bounds for

$$
\begin{aligned}
\mathcal{X}^{h} & \text { in } \mathrm{L}^{\infty}(0, T ; \mathrm{BV}(\Omega)), \\
u^{h} & \text { in } \mathrm{L}^{2}\left(0, T ; \mathrm{H}^{1,2}(\Omega)\right), \\
u^{h} & \text { in } \mathrm{L}^{\infty}\left(0, T ; \mathrm{L}^{p}(\Omega)\right) \text { for all } 1 \leqslant p<\infty, \\
\partial_{t}^{-h}\left(u^{h}+\mathcal{X}^{h}\right) & \text { in } \mathrm{L}^{2}\left(0, T ; \mathrm{H}^{-1,2}(\Omega)\right) .
\end{aligned}
$$

Proof. See [11].

Moreover an estimate for time differences can be derived, which is crucial for the $\mathrm{L}^{1}\left(\Omega_{T}\right)$ compactness of $u^{h}$ and $\mathcal{X}^{h}$ :

LEMma 4.8 For any $0<\tau<T$ we have

$$
\int_{0}^{T} \int_{\Omega}\left(\left|u_{t}^{h}-u_{t-\tau}^{h}\right|+\mid \mathcal{X}_{t}^{h}-\mathcal{X}_{t-\tau}^{h}\right) \mid \mathrm{d} \mathcal{L}^{3} \mathrm{~d} t \leqslant C \tau^{1 / 3}
$$

uniformly in $h>0$.

Proof. For the proof see [11]. 
REMARK 4.9 It is this lemma which requires the assumption $\mathcal{H}^{2}\left(\Gamma_{\mathrm{D}}\right) \neq 0$. In [11] the assertion is also proved for a pure homogeneous Neumann condition but here the global minimising property of the functions $\mathcal{X}_{t}^{h}$ is used, in particular to exclude oscillations between the states $\mathcal{X}_{t}^{h}$ and $1-\mathcal{X}_{t}^{h}$.

\section{Convergence to solutions}

To prove Theorem 1.2 the passage to a limit in the approximate Gibbs-Thomson equations (4.13) is crucial. If a loss of surface mass for the phase interfaces can be excluded as in [11], a lemma of Reshetnyak [14] proves convergence of the Gibbs-Thomson law within the BV-formulation. In contrast, local minimisation requires different arguments. We deal with the surface measures $\left|\nabla \mathcal{X}_{t}^{h}\right|$ and apply a convergence result for surfaces with mean curvature given by a Sobolev function in the ambient space ([17]; see Theorem A.2 in the appendix).

LEMMA 5.1 There are functions

$$
\begin{gathered}
\mathcal{X} \in \mathrm{L}^{\infty}(0, T ; \operatorname{BV}(\Omega ;\{0,1\})), \\
u \in \mathrm{L}^{2}\left(0, T ; u_{\mathrm{D}}+M_{0}\right) \cap \mathrm{L}^{\infty}\left(0, T ; \mathrm{L}^{p}(\Omega)\right) \quad \text { for all } 1 \leqslant p<\infty,
\end{gathered}
$$

and a subsequence $h \rightarrow 0$, such that for all $1 \leqslant p<\infty$,

$$
\begin{aligned}
& \mathcal{X}^{h} \rightarrow \mathcal{X}, \quad u^{h} \rightarrow u \quad \text { in } \mathrm{L}^{p}\left(\Omega_{T}\right), \\
& \mathcal{X}^{h}(t) \rightarrow \mathcal{X}(t), \quad u^{h}(t) \rightarrow u(t) \quad \text { in } \mathrm{L}^{p}(\Omega) \text { for almost all } t \in(0, T), \\
& u^{h} \rightarrow u \quad \text { weakly in } \mathrm{L}^{2}\left(0, T ; \mathrm{H}^{1,2}(\Omega)\right) \text {. }
\end{aligned}
$$

Proof. The compactness in $\mathrm{L}^{p}\left(\Omega_{T}\right)$ is obtained from the theorem of Fréchet-KolmogorovM. Riesz and equations $4.15,4$, 4.17). This yields 5.2 and together with $\mathrm{L}^{2}\left(0, T ; \mathrm{H}^{1,2}(\Omega)\right)$ being reflexive and (4.16) the assertion (5.3).

From Lemma 5.1 and (4.12) it is straightforward to show that $(u, \mathcal{X})$ solves the energy-balance equation (1.1) in the sense of distributions. To turn to the Gibbs-Thomson law let $(u, \mathcal{X})$ be as in Lemma 5.1 and $h \rightarrow 0$ be a subsequence for which 5.1 - 5.3 hold. We set

$$
E(t):=\{\mathcal{X}(t)=1\}, \quad v(t):= \begin{cases}\nabla \mathcal{X}(t) /|\nabla \mathcal{X}(t)| & \text { on } \partial^{*} E(t), \\ 0 & \text { otherwise, }\end{cases}
$$

and define integral 2-varifolds $\mu_{t}^{h}$ on $\Omega$ by

$$
\mu_{t}^{h}(\eta)=\int_{\Omega} \eta\left|\nabla \mathcal{X}_{t}^{h}\right| \quad \text { for } \eta \in C_{\mathrm{c}}^{0}(\Omega)
$$

We complete the proof of Theorem 1.2 .

THEOREM 5.2 For almost all $t \in(0, T)$ there exists an integral 2-varifold $\mu_{t}$ with locally bounded first variation and mean curvature vector $\vec{H}_{\mu_{t}} \in \mathrm{L}_{\mathrm{loc}}^{4}\left(\mu_{t}\right)$ such that

$$
\begin{gathered}
\quad \partial^{*} E(t) \subset \operatorname{spt}\left(\mu_{t}\right) \cap\left\{\theta^{2}\left(\mu_{t}\right) \text { odd }\right\}, \\
\vec{H}_{\mu_{t}}=u(t) v(t) \quad \mu_{t} \text {-almost everywhere in } \Omega .
\end{gathered}
$$


In particular $\partial^{*} E(t)$ has for almost all $t \in(0, T)$ a generalised mean curvature vector according to Definition 1.1 and the Gibbs-Thomson law (1.5) is satisfied. The integral varifolds $\mu_{t}$ can be obtained as limit points of $\left(\mu_{t}^{h}\right)_{h>0}$.

Proof. We restrict ourselves to points $t \in(0, T)$ for which 5.2 holds. By 4.16 and the Fatou lemma we deduce

$$
\left(t \mapsto \liminf _{h \rightarrow 0}\left\|u^{h}(t, \cdot)\right\|_{\mathrm{H}^{1,2}(\Omega)}\right) \in \mathrm{L}^{2}(0, T) .
$$

In consequence for almost all $t \in(0, T)$ there exists a subsequence $h_{i}(t) \rightarrow 0(i \rightarrow \infty)$ and a function $v \in \mathrm{H}^{1,2}(\Omega)$ with

$$
u_{t}^{h_{i}} \rightarrow v \quad \text { weakly in } \mathrm{H}^{1,2}(\Omega) .
$$

The Rellich-Kondrashov embedding theorem (see [5, Theorem 5.7.1]) and 5.2) give $v=u(t)$. Observing that $\left\|h K_{0}^{h}(f)\right\|_{\mathrm{H}^{1,2}(\Omega)} \rightarrow 0$ we get

$$
u_{t}^{h_{i}}+h_{i} K_{0}^{h_{i}}(f) \rightarrow u(t) \quad \text { weakly in } \mathrm{H}^{1,2}(\Omega) .
$$

Moreover by 4.15 the functions $\mathcal{X}_{t}^{h_{i}}$ are uniformly bounded in $\operatorname{BV}(\Omega)$ for almost all $t \in(0, T)$. By the weak*-compactness of Radon measures (see [19, Theorem 4.4]) we can choose a further subsequence of $h_{i} \rightarrow 0$ to obtain for almost all $t \in(0, T)$ Radon measures $V_{t}$ on $G^{2} \Omega$ with

$$
V_{\mu_{t}^{h_{i}}} \stackrel{*}{\rightarrow} V_{t} \quad \text { in } C_{\mathrm{c}}^{0}\left(G^{2} \Omega\right)^{*} .
$$

Moreover the mean curvature equation 4.13 becomes

$$
\vec{H}_{\mu_{t}^{h_{i}}}=\left(u_{t}^{h_{i}}+h_{i} K_{0}^{h_{i}}(f)\right) v_{t}^{h_{i}} .
$$

By (5.2), (5.4), (5.5) and (5.6) all assumptions of the convergence theorem in [17] (see Theorem A.2 in the appendix) are fulfilled and we conclude that there is an integral 2-varifold $\mu_{t}$ on $\Omega$ such that

$$
V_{t}=V_{\mu_{t}}, \quad \mu_{t}^{h_{i}} \stackrel{*}{\rightarrow} \mu_{t} \quad \text { in } C_{\mathrm{c}}^{0}(\Omega)^{*} .
$$

Moreover $\partial^{*} E(t) \subset \operatorname{spt}\left(\mu_{t}\right)$ and $\mu_{t}$ has locally bounded first variation with mean curvature vector

$$
\vec{H}_{\mu_{t}} \in \mathrm{L}_{\mathrm{loc}}^{4}\left(\mu_{t}\right),
$$

which satisfies the Gibbs-Thomson law

$$
\vec{H}_{\mu_{t}}=u(t) v(t)
$$

$\mu_{t}$-almost everywhere. In view of [17, Theorem 1.2] and [6, 5.7 Lemma 2] we obtain

$$
\partial^{*} E(t) \subset\left\{\theta^{2}\left(\mu_{t}, \cdot\right) \text { odd }\right\},
$$

which completes the proof.

In Theorem 5.2 the location of the hidden boundaries $\operatorname{spt}\left(\mu_{t}\right) \backslash \partial^{*} E_{t}$ remains dependent on the choice of subsequences in (5.5), which itself depends on time $t \in(0, T)$. This fact is the cause of bad control over the hidden boundaries and motivates the definition of mean curvature for the phase interface $\partial^{*}\{\mathcal{X}(t)=1\}$. 


\section{Behaviour of solutions}

We compare solutions by local minimisation and solutions by global minimisation. First we state some special properties of solutions by global minimisation.

REMARK 6.1 The time-discretisation in [11] admits an additional penalisation term and minimises for $\Lambda>0$ the functionals

$$
F_{t}^{\Lambda, h}(\mathcal{X})=F_{t}^{h}(\mathcal{X})+\Lambda \int_{\Omega} K_{0}^{h}\left(\mathcal{X}-\mathcal{X}_{t-h}^{h}\right)\left(\mathcal{X}-\mathcal{X}_{t-h}^{h}\right)
$$

Solutions $(u, \mathcal{X})$ of the Stefan problem with Gibbs-Thomson law, which were constructed by a global minimisation of the functionals $F_{t}^{\Lambda, h}$, satisfy for all comparison functions $\psi \in$ $\mathrm{L}^{\infty}(0, T ; \mathrm{BV}(\Omega ;\{0,1\}))$ the inequality

$$
\int_{0}^{T} \int_{\Omega}|\nabla \mathcal{X}|-\int_{\Omega_{T}} u \mathcal{X} \leqslant \int_{0}^{T} \int_{\Omega}|\nabla \psi|-\int_{\Omega_{T}} u \psi+\left(\frac{1}{2}+\Lambda\right) \int_{\Omega_{T}}|\mathcal{X}-\psi| .
$$

Therefore for almost all $t \in(0, T)$ the $\mathcal{X}(t)$ are global minimisers of

$$
F_{t}(\tilde{\mathcal{X}})=\int_{\Omega}|\nabla \tilde{\mathcal{X}}|-\int_{\Omega}\left(u+\mathcal{X}-\frac{1}{2}+2 \Lambda\left(\mathcal{X}-\frac{1}{2}\right)\right)(t) \tilde{\mathcal{X}}
$$

on $\operatorname{BV}(\Omega ;\{0,1\})$. This leads to additional regularity properties. From 4.17$)$ we obtain as in Remark 4.3 for almost all $t \in(0, T)$, all $\overline{B_{r}(x)} \subset \Omega$ and $\tilde{\mathcal{X}} \in \mathrm{BV}(\Omega ;\{0,1\})$ with $\tilde{\mathcal{X}}=\mathcal{X}(t)$ in $\Omega \backslash \overline{B_{r}(x)}$ the estimate

$$
\int_{\Omega}|\nabla \mathcal{X}(t)|-\int_{\Omega}|\nabla \tilde{\mathcal{X}}| \leqslant\left(\|u\|_{\mathrm{L}^{\infty}\left(0, T ; \mathrm{L}^{p}(\Omega)\right)}+C_{\Lambda, \Omega, p}\right)\left(\omega_{3} r^{3}\right)^{1-1 / p} .
$$

This shows that $\partial^{*}\{\mathcal{X}(t)=1\}$ has a $C^{1, \alpha}$-almost-minimal boundary for all $0<\alpha<1 / 2$. After changing $\mathcal{X}(t)$ on a set of $\mathcal{L}^{3}$-measure zero, $\partial\{\mathcal{X}(t)=1\}$ is a $C^{1, \alpha}$-surface. Moreover a theorem of Tamanini ([21], see [22, Theorem XI.8.5]) gives the existence of $r_{0}>0$ and $0<c_{0}<1$, independent of $t \in(0, T)$, such that for all $0<r<r_{0}$ and all $x \in \partial\{\mathcal{X}(t)=1\}$,

$$
c_{0} \leqslant \frac{\mathcal{L}^{3}\left(\{\mathcal{X}(t)=1\} \cap B_{r}(x)\right)}{\omega_{3} r^{3}} \leqslant 1-c_{0} .
$$

This regularity influences the behaviour of the solutions by global minimisation as we will see in the following example.

\subsection{Example}

Consider two ice balls in a container of water. Do the balls touch continuously when they grow monotonically in time? For solutions by global minimisation inequality (6.3) gives a first answer: If the balls would touch as $t \uparrow t_{0}$ one finds $\varepsilon>0$ and for all $t \in\left[t_{0}-\varepsilon, t_{0}\right]$ points contradicting 6.3. Therefore solutions by global minimising create bridge-like connections at a positive distance. To investigate this situation let $R, L$ with $0<R<L / 2$ be given, set $\Omega=B_{L}(0), f=0$ and $\Gamma_{\mathrm{D}}=\emptyset$ (note that this implies $K^{h}=K_{0}^{h}=\tilde{K}^{h}$ ). We choose the pure Neumann condition 
for simplicity although our existence result does not comprise this case. For a Dirichlet or mixed boundary condition we expect a similar behaviour of solutions.

For $r \in(0, R)$ define ball-like solutions $\overline{\mathcal{X}}(r)$ by

$$
E(r):=B_{r}\left(x_{-}\right) \cup B_{r}\left(x_{+}\right), \quad \overline{\mathcal{X}}(r):=\mathcal{X}_{\Omega \backslash E(r)},
$$

where $x_{-}=(0,0,-R)$ and $x_{+}=(0,0, R)$ denote the centres of the ice balls.

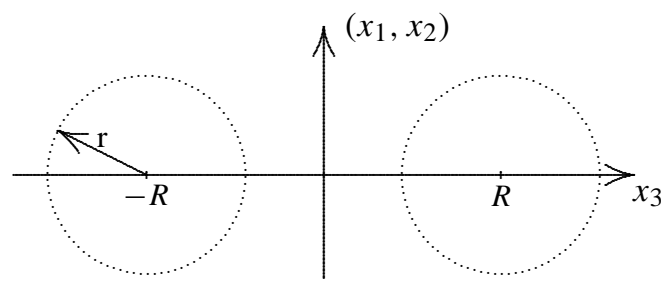

Choose an initial radius $r_{0} \in(0, R)$ and an initial temperature $u_{0} \in \mathrm{H}^{1,2}(\Omega) \cap \mathrm{L}^{\infty}(\Omega)$ such that the ice balls have to grow monotonically (without proof we assume that this is possible).

For $r \in(0, r), \sigma \in[0, r]$ define bridge-like solutions $\psi(r, \sigma)$ by $S_{\sigma}:=Z_{\sigma, R}(0), \psi(r, \sigma):=$ $\mathcal{X}_{\Omega \backslash\left(E(r) \cup S_{\sigma}\right)}$.

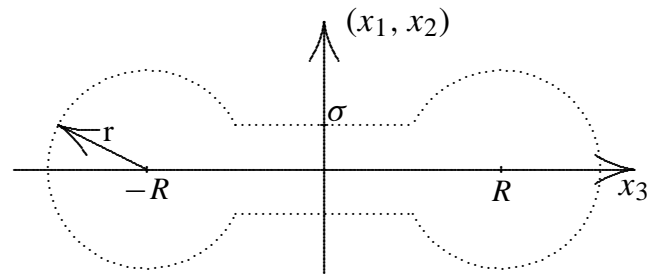

We expect minimisers of the functionals $F_{t}^{h}$ and $F_{i}$ to be ball- or bridge-like, when ball-like solutions are preferred to bridge-like solutions or vice versa. To analyse qualitatively the behaviour of solutions we restrict ourselves to the class of functions $\overline{\mathcal{X}}(r)$ and $\psi(r, \sigma)$, when minimising the respective time-discrete functionals. For a time step $h>0$ we assume ball-like solutions until a time $t \in(0, T)$, so $\mathcal{X}_{\tau}^{h}=\overline{\mathcal{X}}\left(r_{\tau}^{h}\right)$ for $\tau \leqslant t, r_{\tau}^{h}$ increasing in $\tau$. In [11], $\mathcal{X}_{t+h}^{h}$ is chosen to be a global minimiser of

$$
F_{t+h}^{h}(\mathcal{X})=\int_{\Omega}|\nabla \mathcal{X}|-\int_{\Omega}\left(K^{h}\left(u_{t}^{h}\right) \mathcal{X}-\frac{1}{2} K^{h}\left(\mathcal{X}-\overline{\mathcal{X}}\left(r_{t}^{h}\right)\right)\left(\mathcal{X}-\overline{\mathcal{X}}\left(r_{t}^{h}\right)\right)\right) .
$$

We claim that for all radii near $r=R$ the global minimisation prefers bridge-like solutions to balllike solutions. The bridges must have a minimal diameter. Furthermore in case their diameters are not large enough ball-like solutions are preferred.

PROPOSITION 6.2 Using the global minimisation there are $\delta>0$ and $\sigma_{0}>0$ such that for all $h>0, r_{t}^{h} \in(0, R)$ and $r \in(R-\delta, R), r>r_{t}^{h}$, we have

$$
\min _{\sigma \in[0, r]} F_{t+h}^{h}(\psi(r, \sigma))<\min _{\sigma \in\left[0, \sigma_{0}\right]} F_{t+h}^{h}(\psi(r, \sigma)) \leqslant F_{t+h}^{h}(\overline{\mathcal{X}}(r)) .
$$


For each such $r$ there is a constant $\sigma_{1}>0$ such that for all $0<\sigma<\min \left(\sigma_{1}, \frac{1}{3}(R-r)\right)$,

$$
F_{t+h}^{h}(\overline{\mathcal{X}}(r))-F_{t+h}^{h}(\psi(r, \sigma))<0 .
$$

Proof. Consider

$$
\begin{aligned}
d(r, \sigma)= & d_{t+h}^{h}(r, \sigma) \\
= & F_{t+h}^{h}(\overline{\mathcal{X}}(r))-F_{t+h}^{h}(\psi(r, \sigma)) \\
= & 4 \pi\left(r^{2}-r \sqrt{r^{2}-\sigma^{2}}-\left(R-\sqrt{r^{2}-\sigma^{2}}\right) \sigma\right)-\int_{\Omega} K^{h}\left(u_{t}^{h}\right) \mathcal{X}_{S(\sigma) \backslash E(r)} \\
& -\frac{1}{2} \int_{\Omega} K^{h}\left(\mathcal{X}_{S(\sigma) \backslash E(r)}\right)\left(2\left(\overline{\mathcal{X}}\left(r_{t}^{h}\right)-\overline{\mathcal{X}}(r)\right)+\mathcal{X}_{S(\sigma) \backslash E(r)}\right) .
\end{aligned}
$$

For the derivative we apply the coarea formula [6, Theorem 3.4.2] in differential form:

$$
\begin{aligned}
\frac{\partial}{\partial \sigma} d(r, \sigma)= & 4 \pi\left(\sqrt{r^{2}-\sigma^{2}}+\sigma \frac{\sqrt{r-\sigma}}{\sqrt{r+\sigma}}-R\right)-\int_{\Gamma_{r, \sigma}} K^{h}\left(u_{t}^{h}\right)(y) \mathrm{d} \mathcal{H}^{2}(y) \\
& -\int_{\Gamma_{r, \sigma}} K^{h}\left(\mathcal{X}_{S(r) \backslash E(r)}+\overline{\mathcal{X}}\left(r_{t}^{h}\right)-\overline{\mathcal{X}}(r)\right)(y) \mathrm{d} \mathcal{H}^{2}(y),
\end{aligned}
$$

with $\Gamma_{r, \sigma}=\left\{y:\left|\left(y_{1}, y_{2}\right)\right|=\sigma, y_{3} \in\left(-R+\sqrt{r^{2}-\sigma^{2}}, R-\sqrt{r^{2}-\sigma^{2}}\right)\right\}$.

First observe that for $r=R$ a bridge-like solution is preferred:

$$
\max _{\sigma \in[0, R]} d(R, \sigma)>0 .
$$

Next check that the function $d$ is continuous in $r$ and uniformly Hölder continuous in $\sigma$. These properties yield the existence of constants $\delta>0$ and $\sigma_{0}>0$ independent of $h>0$ such that

$$
\max _{\sigma \in[0, r]} d(r, \sigma)>\max _{\sigma \in\left[0, \sigma_{0}\right]} d(r, \sigma) \geqslant 0 .
$$

To prove 6.4 one calculates, for $r>r_{t}^{h}$,

$$
\begin{aligned}
d(r, \sigma) \leqslant & -\mathcal{H}^{2}(\partial(S(\sigma) \backslash E(r)))+2 \mathcal{H}^{2}(\partial E(r) \cap S(\sigma)) \\
& +\left\|K^{h}\left(u_{t}^{h}\right)\right\|_{\mathrm{L}^{4}(\Omega)}|S(\sigma) \backslash E(r)|^{3 / 4} .
\end{aligned}
$$

Using $K^{h}\left(u_{t}^{h}\right)^{3}$ as a test function in the equation $K^{h}\left(u_{t}^{h}\right)-h \Delta K^{h}\left(u_{t}^{h}\right)=u_{t}^{h}$ and recalling (4.17) yields $\left\|K^{h}\left(u_{t}^{h}\right)\right\|_{\mathrm{L}^{4}(\Omega)} \leqslant C$. With the isoperimetrical inequality [6, Theorem 5.6.2] we derive

$$
\begin{aligned}
-\mathcal{H}^{2}(\partial(S(\sigma) \backslash E(r)))+\left\|K^{h}\left(u_{t}^{h}\right)\right\|_{\mathrm{L}^{4}(\Omega)}\left(\mathcal{L}^{3}(S(\sigma) \backslash E(r))\right)^{3 / 4} \\
\quad \leqslant\left(-1+C \mathcal{H}^{2}(\partial(S(\sigma) \backslash E(r)))^{1 / 8}\right) \mathcal{H}^{2}(\partial(S(\sigma) \backslash E(r))) .
\end{aligned}
$$

Now choose $\sigma_{1}>0$ such that for all $r>r_{t}^{h}$ and $\sigma<\sigma_{1}$,

$$
\mathcal{H}^{2}(\partial(S(\sigma) \backslash E(r)))^{1 / 8} \leqslant \frac{1}{2 C},
$$


and obtain

$$
\begin{aligned}
d(r, \sigma) & \leqslant-\frac{1}{2} \mathcal{H}^{2}(\partial(S(\sigma) \backslash E(r)))+2 \mathcal{H}^{2}(\partial E(r) \cap S(\sigma)) \\
& =-\frac{1}{2} \mathcal{H}^{2}(\partial S(\sigma) \backslash E(r))+\frac{3}{2} \mathcal{H}^{2}(\partial E(r) \cap S(\sigma)) \\
& =-\pi\left(R-\sqrt{r^{2}-\sigma^{2}}\right) \sigma+3 \pi r\left(r-\sqrt{r^{2}-\sigma^{2}}\right) \\
& \leqslant-\pi(R-r) \sigma+3 \pi r\left(r-\left(r-\frac{1}{2 r} \sigma^{2}\right)\right) \\
& <-\frac{\pi}{2}(R-r) \sigma
\end{aligned}
$$

for $0<\sigma<\frac{1}{3}(R-r)$.

Restricting to special initial data we show that local minimisation prefers ball-like solutions.

Proposition 6.3 Let initial data $0 \ll r_{0}<R$ and $u_{0} \in \mathrm{H}^{1,2}(\Omega) \cap \mathrm{L}^{\infty}(\Omega)$ with $u_{0}>-1 / 2$ be given. Then solutions by local minimisation do not build bridges before the ice balls touch.

To determine $\mathcal{X}_{t+h}^{h}$ we have to consider the evolution $\left\{\tilde{\mathcal{X}}_{i}\right\}_{i \in \mathbb{N}}$, where $\tilde{\mathcal{X}}_{i}$ minimises the functional

$$
F_{i}(\mathcal{X})=F_{t}^{h}(\mathcal{X})+\lambda_{h} \int_{\Omega} K^{\varepsilon_{h}}\left(\mathcal{X}-\tilde{\mathcal{X}}_{i-1}\right)\left(\mathcal{X}-\tilde{\mathcal{X}}_{i-1}\right)
$$

Assume again $\mathcal{X}_{t}^{h}=\overline{\mathcal{X}}\left(r_{t}^{h}\right)$ and $\tilde{\mathcal{X}}_{i-1}=\overline{\mathcal{X}}\left(\tilde{r}_{i-1}\right)$, and define

$$
\begin{aligned}
\tilde{d}_{i}(r, \sigma) & =F_{i}(\overline{\mathcal{X}}(r))-F_{i}(\psi(r, \sigma)) \\
& =d(r, \sigma)-\lambda_{h} \int_{\Omega} K^{\varepsilon h}\left(\mathcal{X}_{S(\sigma) \backslash E(r)}\right)\left(\mathcal{X}_{S(\sigma) \backslash E(r)}+2 \overline{\mathcal{X}}\left(\tilde{r}_{i-1}\right)-2 \overline{\mathcal{X}}(r)\right) .
\end{aligned}
$$

In a first step we notice that bridge-like solutions are only competitive in a small neighbourhood of $r=R$ shrinking to a point with $h \rightarrow 0$ :

Lemma 6.4 There exists a function $\omega:\left(0, h_{0}\right) \rightarrow \mathbb{R}$ with $\omega(h) \rightarrow 0(h \rightarrow 0)$ such that for all $\tilde{r}_{i-1} \leqslant r<R-\omega(h)$ and $0<\sigma \leqslant r$,

$$
F_{i}(\overline{\mathcal{X}}(r))<F_{i}(\psi(r, \sigma)) .
$$

Proof. By 6.4 we assume $\sigma>\sigma_{1}(r):=\min \left(\sigma_{1}, \frac{1}{3}(R-r)\right)$. Since $d(r, \sigma) \leqslant C(R)$ for all $r, \sigma$ we have

$$
\begin{aligned}
\tilde{d}_{i}(r, \sigma) & \leqslant C(R)-\lambda_{h} \int_{\Omega} K^{\varepsilon_{h}}\left(\mathcal{X}_{S\left(\sigma_{1}(r)\right) \backslash E(r)}\right) \mathcal{X}_{S\left(\sigma_{1}(r)\right) \backslash E(r)} \\
& \leqslant C(R)-\lambda_{h} \int_{\Omega} K^{\varepsilon_{h}}\left(\mathcal{X}_{B_{\sigma_{1}(r)}(0)}\right) \mathcal{X}_{B_{\sigma_{1}(r)}(0)}
\end{aligned}
$$

Considering for $\alpha>0$ and $0<m<L$ the fundamental solution $\Phi_{\alpha}$ of id $-\alpha \Delta$ in $\mathbb{R}^{3}$ we observe that $\nabla\left(\mathcal{X}_{B_{m}(0)} * \Phi_{\alpha}\right) \cdot v_{\partial B_{m}(0)}<0$. The maximum principle theorems 8.1, 8.6 in [20] applied to id $-\alpha \Delta$ yield $K^{\alpha}\left(\mathcal{X}_{B_{m}(0)}\right) \geqslant \mathcal{X}_{B_{m}(0)} * \Phi_{\alpha}$ and

$$
\int_{B_{m}(0)} K^{\alpha}\left(\mathcal{X}_{B_{m}(0)}\right) \geqslant m^{3} \int_{B_{1}(0)} \mathcal{X}_{B_{1}(0)} * \Phi_{\alpha m^{-2}}
$$


Thus

$$
\tilde{d}_{i}(r, \sigma) \leqslant C(R)-\lambda_{h} \sigma_{1}(r)^{3} \int_{B_{1}(0)} \mathcal{X}_{B_{1}(0)} * \Phi_{\varepsilon_{h} \sigma_{1}(r)^{-2}} \leqslant 0
$$

for $r<R-\omega(h)$, if $\omega(h)>0$ is chosen with

$$
\lambda_{h} \sigma_{1}(R-\omega(h))^{3} \rightarrow \infty, \quad \frac{\varepsilon_{h}}{\sigma_{1}(R-\omega(h))^{2}} \rightarrow 0 .
$$

Observing that

$$
\begin{aligned}
\lambda_{h} \sigma_{1}(R-\omega(h))^{3} & \geqslant \min \left\{\lambda_{h} \sigma_{1}^{3}, \frac{1}{27} \lambda_{h} \omega(h)^{3}\right\}, \\
\frac{\varepsilon_{h}}{\sigma_{1}(R-\omega(h))^{2}} & \leqslant \max \left\{\frac{\varepsilon_{h}}{\sigma_{1}^{2}}, \frac{9 \varepsilon_{h}}{\omega(h)^{2}}\right\}
\end{aligned}
$$

we find that this can be satisfied with $\omega(h) \rightarrow 0(h \rightarrow 0)$.

By Lemma 6.4 the creation of a bridge at a positive distance of the balls yields a jump of the radius. This jump affects the first evolution and is never limited to the evolutions $\left\{\tilde{\mathcal{X}}_{i}\right\}_{i \in \mathbb{N}}$.

LEMMA 6.5 If for $t \in(0, T)$ and a subsequence $h \rightarrow 0$ there are integers $\alpha(h)$ such that

$$
\mathcal{X}_{t+\alpha(h) h}^{h}=\overline{\mathcal{X}}\left(r_{t+\alpha(h) h}^{h}\right), \quad \mathcal{X}_{t+\alpha(h) h+h}^{h} \text { is a bridge-like solution, }
$$

then

$$
r_{t+\alpha(h) h}^{h} \rightarrow R \quad(h \rightarrow 0)
$$

Proof. Lemma 6.4 ensures the existence of a sequence $i(h)$ such that

$$
\tilde{\mathcal{X}}_{t+\alpha(h) h, i(h)}^{h}=\overline{\mathcal{X}}\left(\tilde{r}_{t+\alpha(h) h, i(h)}^{h}\right), \quad \tilde{r}_{t+\alpha(h) h, i(h)}^{h}>R-\omega(h) .
$$

Now we set

$$
\tilde{r}_{i}^{h}=\tilde{r}_{t+\alpha(h) h, i(h)}^{h}, \quad \tilde{r}_{0}^{h}=r_{t+\alpha(h) h}^{h} .
$$

If the assertion is false, then for a subsequence $h \rightarrow 0$,

$$
\lim _{h \rightarrow 0}\left(\tilde{r}_{i}^{h}-\tilde{r}_{0}^{h}\right)>0 .
$$

This yields

$$
K^{h}\left(\overline{\mathcal{X}}\left(\tilde{r}_{i}^{h}\right)-\overline{\mathcal{X}}\left(\tilde{r}_{0}^{h}\right)\right) \approx \overline{\mathcal{X}}\left(\tilde{r}_{i}^{h}\right)-\overline{\mathcal{X}}\left(\tilde{r}_{0}^{h}\right) .
$$

In addition $u_{\tau}^{h}=K^{h}\left(u_{\tau-h}^{h}-\left(\mathcal{X}_{\tau}^{h}-\mathcal{X}_{\tau-h}^{h}\right)\right)$, the assumed monotonic growth and invoking the maximum principle twice implies $K^{h}\left(u_{\tau}^{h}\right) \geqslant \inf _{\Omega} u_{0}$ (see the proof of Lemma 4.1). Now we obtain

$$
\begin{aligned}
& 6 \omega_{3}\left(\tilde{r}_{i}^{h}\right)^{2}-6 \omega_{3}\left(\tilde{r}_{0}^{h}\right)^{2}-\int_{\Omega} K^{h}\left(u_{t+\alpha(h) h}^{h}\right)\left(\overline{\mathcal{X}}\left(\tilde{r}_{i}^{h}\right)-\overline{\mathcal{X}}\left(\tilde{r}_{0}^{h}\right)\right) \\
& \quad+\frac{1}{2} \int_{\Omega} K^{h}\left(\overline{\mathcal{X}}\left(\tilde{r}_{i}^{h}\right)-\overline{\mathcal{X}}\left(\tilde{r}_{0}^{h}\right)\right)\left(\overline{\mathcal{X}}\left(\tilde{r}_{i}^{h}\right)-\overline{\mathcal{X}}\left(\tilde{r}_{0}^{h}\right)\right)>\int_{\Omega}\left(\inf _{\Omega} u_{0}+\frac{1}{2}\right)\left|\overline{\mathcal{X}}\left(\tilde{r}_{i}^{h}\right)-\overline{\mathcal{X}}\left(\tilde{r}_{0}^{h}\right)\right|>0
\end{aligned}
$$

since $\inf _{\Omega} u_{0}>-1 / 2$. This contradicts $F_{t+\alpha(h) h}^{h}\left(\overline{\mathcal{X}}\left(\tilde{r}_{i}^{h}\right)\right) \leqslant F_{t+\alpha(h) h}^{h}\left(\overline{\mathcal{X}}\left(\tilde{r}_{0}^{h}\right)\right)$. 
Now we are able to prove Proposition 6.3. Notice that $u+\mathcal{X}$ is in $C^{0}\left(0, T ; \mathrm{L}^{p}(\Omega)\right)$ for all $1 \leqslant p$ $<\infty$ (see [11], [12]), and that for $\Gamma_{\mathrm{D}}=\emptyset, f=0$ the energies are monotone, that is,

$$
t \mapsto \int_{\Omega}|\nabla \mathcal{X}(t)|+\frac{1}{2} \int_{\Omega} u(t)^{2}, \quad t \mapsto \int_{\Omega}\left|\nabla \mathcal{X}_{t}^{h}\right|+\frac{1}{2} \int_{\Omega}\left(u_{t}^{h}\right)^{2}
$$

are decreasing in $(0, T)$.

To sketch the idea consider a single ball jumping at a time $t$, that is,

$$
r(t+)-r(t-)=\lim _{\delta \rightarrow 0}(r(t+\delta)-r(t-\delta))>0 .
$$

The continuity of $u+\mathcal{X}$ forces $u$ to jump as well:

$$
u(t+)=u(t-)-(\mathcal{X}(t+)-\mathcal{X}(t-)) .
$$

The maximum principle implies $u(t-) \geqslant \inf _{\Omega} u_{0}>-1 / 2$ but in contradiction to the monotonicity of energy we get

$$
3 \omega_{3}\left(r(t+)^{2}-r(t-)^{2}\right)+\frac{1}{2} \int_{\Omega}\left(u(t+)^{2}-u(t-)^{2}\right)>0 .
$$

To adapt this idea notice that by Lemma 6.4 the radii have to jump when a bridge-like connection appears at a positive distance of the balls. With the aid of Lemma 6.5 we derive a contradiction to the monotonicity of energy at the level of the time-discrete evolutions $\mathcal{X}^{h}$.

Applying the $\mathrm{L}^{p}(\Omega)$-convergence of $u_{t}^{h}, \mathcal{X}_{t}^{h}$ for a subsequence $h \rightarrow 0$ and almost all $t \in(0, T)$ and using the fact that $u+\mathcal{X} \in C^{0}\left(0, T ; \mathrm{L}^{p}(\Omega)\right)$ we restrict ourselves to the following situation:

ASSUMPTION There are subsequences $h \searrow 0$ and $\delta \searrow 0$ such that

$$
\begin{aligned}
& \mathcal{X}_{t \pm \delta}^{h} \stackrel{h \rightarrow 0}{\rightarrow} \mathcal{X}(t \pm \delta) \quad \text { in } \mathrm{L}^{p}(\Omega), \\
& u_{t \pm \delta}^{h} \stackrel{h \rightarrow 0}{\rightarrow} u(t \pm \delta) \quad \text { in } \mathrm{L}^{p}(\Omega), \\
& \mathcal{X}(t-\delta) \rightarrow \mathcal{X}(t-), \quad \mathcal{X}(t+\delta) \rightarrow \mathcal{X}(t+) \quad \text { in } \mathrm{L}^{p}(\Omega), \\
& \mathcal{X}_{t-\delta}^{h}=\overline{\mathcal{X}}\left(r_{t-\delta}^{h}\right), \quad \mathcal{X}(t-\delta)=\overline{\mathcal{X}}(r(t-\delta)), \quad r(t-\delta) \rightarrow r(t-)<R .
\end{aligned}
$$

(In case of $r(t-)=R$ we are done.)

Claim There exists $\delta_{0}>0$ with

$$
\begin{aligned}
\mathcal{X}(t+\delta) & =\overline{\mathcal{X}}(r(t+\delta)) \quad \text { for all } \delta<\delta_{0}, \\
r(t+\delta) & \rightarrow r(t-) .
\end{aligned}
$$

Assuming this claim to be false we obtain a subsequence $\delta \rightarrow 0$ and $h_{\delta}>0$ such that either

$$
\begin{gathered}
\mathcal{X}_{t+\delta}^{h} \text { is a bridge-like solution for all } h<h_{\delta}, \text { or } \\
r(t+)-r(t-)=\lim _{\delta \rightarrow 0} \lim _{h \rightarrow 0}\left(r_{t+\delta}^{h}-r_{t-\delta}^{h}\right)>0 .
\end{gathered}
$$

In the first case the last two lemmas imply the existence of $\alpha_{\delta}^{h} \leqslant \delta$ such that

$$
\mathcal{X}_{t+\alpha_{\delta}^{h}}^{h}=\overline{\mathcal{X}}\left(r_{t+\alpha_{\delta}^{h}}^{h}\right) \quad \text { for all } h<h_{\delta}, \quad \lim _{\delta \rightarrow 0} \lim _{h \rightarrow 0}\left(r_{t+\alpha_{\delta}^{h}}^{h}-r_{t-\delta}^{h}\right)=R-r(t-)>0 .
$$


So we can prove both cases analogously. We consider the first one. By 4 .1 for $N=[\delta / h]+\left[\alpha_{\delta}^{h} / h\right]$ we obtain

$$
u_{t+\alpha_{\delta}^{h}}^{h}=\left(K^{h}\right)^{N}\left(u_{t-\delta}^{h}\right)-\sum_{j=1}^{N}\left(K^{h}\right)^{N-j+1}\left(\mathcal{X}_{t-\delta+j h}^{h}-\mathcal{X}_{t-\delta+(j-1) h}^{h}\right) .
$$

To estimate $\int_{\Omega}\left(u_{t+\alpha_{\delta}^{h}}^{h}\right)^{2}-\int_{\Omega}\left(u_{t-\delta}^{h}\right)^{2}$ consider a $L^{2}(\Omega)$-orthonormal basis $\left\{w_{k}\right\}_{k \in \mathbb{N}_{0}}$ of eigenfunctions

$$
\begin{gathered}
-\Delta w_{k}=\mu_{k} w_{k} \quad \text { in } \Omega, \quad \nabla w_{k} \cdot v_{\Omega}=0 \quad \text { on } \partial \Omega, \\
w_{0}=\frac{1}{|\Omega|}, \quad \mu_{k} \geqslant 0, \quad \mu_{k} \rightarrow \infty \quad(k \rightarrow \infty) .
\end{gathered}
$$

Since $K^{h}$ is selfadjoint and $K^{h}\left(w_{k}\right)=\left(1+h \mu_{k}\right)^{-1} w_{k}$ we find

$$
\begin{aligned}
\left|\int_{\Omega}\left(K^{h}\right)^{N}\left(u_{t-\delta}^{h}\right)^{2}-\int_{\Omega}\left(u_{t-\delta}^{h}\right)^{2}\right| & =\left|\sum_{k}\left(\left(1+h \mu_{k}\right)^{-2 N}-1\right)\left(\int_{\Omega} u_{t-\delta}^{h} w_{k}\right)^{2}\right| \\
& \geqslant \sum_{k}\left(1-e^{-\frac{4 \delta}{h} \ln \left(1+h \mu_{k}\right)}\right)\left(\int_{\Omega} u_{t-\delta}^{h} w_{k}\right)^{2} \\
& \stackrel{h \rightarrow 0}{\rightarrow} \sum_{k}\left(1-e^{-4 \delta \mu_{k}}\right)\left(\int_{\Omega} u(t-\delta) w_{k}\right)^{2} \\
& \stackrel{\delta \rightarrow 0}{\rightarrow} 0
\end{aligned}
$$

$\left(\left|\int_{\Omega} u_{t-\delta}^{h} w_{k}\right|^{2}\right.$ and $\left|\int_{\Omega} u(t-\delta) w_{k}\right|^{2}$ are convergent majorants).

The monotonic increase of the radius and the maximum principle yield as above the estimate $\left(K^{h}\right)^{N}\left(u_{t-\delta}^{h}\right) \geqslant \inf _{\Omega} u_{0}$ and together with the homogeneous Neumann data we obtain

$$
\begin{aligned}
\lim _{\delta \rightarrow 0} \lim _{h \rightarrow 0}-2 \int_{\Omega}\left(K^{h}\right)^{N}\left(u_{t-\delta}^{h}\right) \cdot \sum_{j=1}^{N}\left(K^{h}\right)^{N-j+1}\left(\mathcal{X}_{t-\delta+j h}^{h}-\mathcal{X}_{t-\delta+(j-1) h}^{h}\right) & \\
& \geqslant 2\left(\inf _{\Omega} u_{0}\right) \int_{\Omega}|\overline{\mathcal{X}}(R)-\mathcal{X}(t-)| .
\end{aligned}
$$

Similar to the calculations above we derive

$$
\int_{\Omega}\left|\sum_{j=1}^{N}\left(K^{h}\right)^{N-j+1}\left(\mathcal{X}_{t-\delta+j h}^{h}-\mathcal{X}_{t-\delta+(j-1) h}^{h}\right)\right|^{2}=\sum_{k}\left|\int_{\Omega} g_{k}^{h, \delta}\left(\mathcal{X}_{t+\alpha}^{h}-\mathcal{X}_{t-\delta}^{h}\right) w_{k}\right|^{2},
$$

with a function $g_{k}^{h, \delta}$ on $\Omega$,

$$
\begin{aligned}
g_{k}^{h, \delta}\left(\mathcal{X}_{t+\alpha}^{h}-\mathcal{X}_{t-\delta}^{h}\right)= & \sum_{j=1}^{N}\left(1+h \mu_{k}\right)^{-N+j-1}\left(\mathcal{X}_{t-\delta+j h}^{h}-\mathcal{X}_{t-\delta+(j-1) h}^{h}\right), \\
& \left(1+h \mu_{k}\right)^{-N} \leqslant g_{k}^{h, \delta} \leqslant 1 .
\end{aligned}
$$


For a subsequence $h \rightarrow 0$ we obtain

$$
g_{k}^{h, \delta}\left(\mathcal{X}_{t+\alpha}^{h}-\mathcal{X}_{t-\delta}^{h}\right) \rightarrow g_{k}^{\delta}(\overline{\mathcal{X}}(R)-\mathcal{X}(t-\delta)) \quad \text { weakly in } \mathrm{L}^{2}(\Omega)
$$

with $e^{-2 \delta \mu_{k}} \leqslant g_{k}^{\delta} \leqslant 1$ and

$$
\begin{aligned}
\liminf _{h \rightarrow 0} \int_{\Omega}\left|\sum_{j=1}^{N}\left(K^{h}\right)^{N-j+1}\left(\mathcal{X}_{t-\delta+j h}^{h}-\mathcal{X}_{t-\delta+(j-1) h}^{h}\right)\right|^{2} \\
\geqslant \sum_{k}\left|\int_{\Omega} g_{k}^{\delta}(\overline{\mathcal{X}}(R)-\mathcal{X}(t-\delta)) w_{k}\right|^{2} \\
\stackrel{\delta \rightarrow 00}{\rightarrow} \sum_{k}\left|\int_{\Omega}(\overline{\mathcal{X}}(R)-\mathcal{X}(t-)) w_{k}\right|^{2}=\int_{\Omega}|\overline{\mathcal{X}}(R)-\mathcal{X}(t-)| .
\end{aligned}
$$

Altogether from (6.6)-6.9) we conclude that

$$
\lim _{\delta \rightarrow 0} \liminf _{h \rightarrow 0} \int_{\Omega}\left(\left(u_{t+\alpha_{\delta}^{h}}^{h}\right)^{2}-\left(u_{t-\delta}^{h}\right)^{2}\right) \geqslant 2\left(\inf _{\Omega} u_{0}\right) \int_{\Omega}|\overline{\mathcal{X}}(R)-\mathcal{X}(t-)|>0
$$

and finally for $u_{0}>-1 / 2$, using the monotonicity of energy,

$$
\begin{aligned}
0 & \geqslant \lim _{\delta \rightarrow 0} \liminf _{h \rightarrow 0}\left[6 \omega_{3}\left(r_{t+\alpha_{\delta}^{h}}^{h}\right)^{2}-6 \omega_{3}\left(r_{t-\delta}^{h}\right)^{2}+\frac{1}{2} \int_{\Omega}\left(\left(u_{t+\alpha_{\delta}^{h}}^{h}\right)^{2}-\left(u_{t-\delta}^{h}\right)^{2}\right)\right] \\
& \left.\geqslant 6 \omega_{3}\left(R^{2}-r(t-)^{2}\right)+\inf _{\Omega} u_{0}\right) \int_{\Omega}|\overline{\mathcal{X}}(R)-\mathcal{X}(t-)|+\frac{1}{2} \int_{\Omega}|\overline{\mathcal{X}}(R)-\mathcal{X}(t-)| \\
& >0 .
\end{aligned}
$$

This is a contradiction.

\section{A. Lipschitz approximation and convergence of mean curvature}

First we state a version of the Lipschitz approximation theorem of Brakke [4, Theorem 5.4] (see also [18]), which we use in Section 3 . Second we quote the convergence result of Schätzle [17], which is crucial in Section 5 .

\section{A.1 Lipschitz approximation}

For an integral ( $n-1)$-varifold $\mu$ in $\Omega \subset \mathbb{R}^{n}$ open, $x_{0} \in \Omega, \varrho>0$ with $B_{\varrho}^{n}\left(x_{0}\right) \subset \Omega$ and an ( $n-1)$-dimensional subspace $T \subset \mathbb{R}^{n}$ we define the tilt, tilt-excess and Lipschitz approximation constant by

$$
\begin{aligned}
\operatorname{tilt}_{\mu}\left(x_{0}, \varrho, T\right) & =\varrho^{-n-1} \int_{B_{\varrho}^{n}\left(x_{0}\right)} \operatorname{dist}\left(x-x_{0}, T\right)^{2} \mathrm{~d} \mu(x), \\
\operatorname{tiltex}_{\mu}\left(x_{0}, \varrho, T\right) & =\varrho^{-n+1} \int_{B_{\varrho}^{n}\left(x_{0}\right)}\left\|T_{x} \mu-T\right\|^{2} \mathrm{~d} \mu(x), \\
\operatorname{lipapp}_{\mu}\left(x_{0}, \varrho, T\right) & =\operatorname{tilt}_{\mu}\left(x_{0}, \varrho, T\right)+\operatorname{tiltex}_{\mu}\left(x_{0}, \varrho, T\right)+\varrho^{3-n} \int_{B_{\varrho}^{n}\left(x_{0}\right)}\left|\vec{H}_{\mu}\right|^{2} \mathrm{~d} \mu,
\end{aligned}
$$

where we set $\operatorname{lipapp}_{\mu}\left(x_{0}, \varrho, T\right)=\infty$ if $\vec{H}_{\mu} \notin \mathrm{L}^{2}\left(\mu\left\llcorner B_{\varrho}^{n}\left(x_{0}\right)\right)\right.$. 
We state here a simplified version of the tilted Lipschitz approximation theorem given in [18], which itself follows from the Lipschitz approximation theorem of Brakke [4, Theorem 5.4]. Roughly speaking, an integral $(n-1)$-varifold, well approximated (with respect to the Lipschitz approximation constant) by a hyperplane $T_{0}$, is for any hyperplane $T$ not vertical to $T_{0}$ given as union of Lipschitz graphs over $T$ :

THEOREM A.1 Let $\mu$ be an integral $(n-1)$-varifold in $B_{7}^{n}(0), \theta_{0} \in \mathbb{N}$ and $T_{0}=\left\{v_{0}\right\}^{\perp}$ be an $(n-1)$-dimensional subspace of $\mathbb{R}^{n}$ with

$$
\left|\vec{e}_{n} \cdot v_{0}\right| \geqslant \lambda>0 .
$$

Let $T_{0}$ be the graph over $\mathbb{R}^{n-1} \times\{0\}$ of a linear map $\mathcal{T}_{0}: \mathbb{R}^{n-1} \rightarrow \mathbb{R}$ :

$$
\left(y, \mathcal{T}_{0} y\right) \in T_{0} \quad \text { for all } y \in \mathbb{R}^{n-1} .
$$

If

$$
\begin{gathered}
\mu\left(B_{7}^{n}(0)\right) \leqslant \Gamma, \quad \mu\left(B_{3}^{n}(0)\right) \leqslant\left(\theta_{0}+1 / 2\right) 3^{n} \omega_{n}, \\
\left(\theta_{0}-1 / 2\right) \omega_{n} \leqslant \mu\left(B_{1}^{n}(0)\right), \quad \operatorname{lipapp}_{\mu}\left(0,7, T_{0}\right) \leqslant \varepsilon,
\end{gathered}
$$

then there exist constants $\delta_{0}>0$ and $c, C>0$ depending on $\Gamma, \lambda, n$ and $\theta_{0}$, and a function $\omega: \mathbb{R} \rightarrow \mathbb{R}$ with $\omega(s) \rightarrow 0(s \rightarrow 0)$ and $\theta_{0}$ Lipschitz continuous maps

$$
f_{i}: B_{\delta_{0}}^{n-1}(0) \rightarrow \mathbb{R}, \quad i=1, \ldots, \theta_{0},
$$

with

$$
\operatorname{Lip}\left(f_{i}\right) \leqslant c, \quad\left\|f_{i}-\mathcal{T}_{0}\right\|_{\mathrm{L}^{\infty}\left(B_{\delta_{0}}^{n-1}(0)\right)} \leqslant \omega(\varepsilon),
$$

such that the following assertions hold:

The set $Y_{0} \subset B_{\delta_{0}}^{n-1}(0)$ consisting of points $y \in B_{\delta_{0}}^{n-1}(0)$ with

$$
\theta^{n-1}(\mu,(y, t))=\#\left\{i: f_{i}(y)=t\right\} \quad \text { for all }-1 / 2<t<1 / 2,
$$

and the set

$$
X_{0}:=\operatorname{spt}(\mu) \cap\left(Y_{0} \times(-1 / 2,1 / 2)\right)=\left\{\left(y, f_{i}(y)\right): y \in Y_{0}, 1 \leqslant i \leqslant \theta_{0}\right\}
$$

satisfy the estimate

$$
\mu\left(\left(B_{\delta_{0}}^{n-1}(0) \times(-1 / 2,1 / 2)\right) \backslash X_{0}\right)+\mathcal{L}^{n-1}\left(B_{\delta_{0}}^{n-1}(0) \backslash Y_{0}\right) \leqslant C \varepsilon .
$$

\section{A.2 Convergence of mean curvatures given as a trace}

Assumption Let $\Omega \subset \mathbb{R}^{n}$ be open, $n \geqslant 2$. For $j \in \mathbb{N}$ let $E_{j} \subset \Omega$ be subsets of finite perimeter and define

$$
\mu_{j}=\left|\nabla \mathcal{X}_{E_{j}}\right|, \quad v_{j}=\frac{\nabla \mathcal{X}_{E_{j}}}{\left|\nabla \mathcal{X}_{E_{j}}\right|} \quad \text { on } \partial^{*} E_{j}
$$


Suppose each integral $(n-1)$-varifold $\mu_{j}$ has a mean curvature vector $\vec{H}_{\mu_{j}}$ given by the trace of a function $u_{j} \in \mathrm{H}^{1, p}(\Omega), n / 2<p<n$, which means that

$$
\vec{H}_{\mu_{j}}=u_{j} v_{j} \quad \text { on } \partial^{*} E_{j}
$$

in the weak sense of

$$
\int_{\Omega}\left(\nabla \eta-\frac{\nabla \mathcal{X}_{E_{j}}}{\left|\nabla \mathcal{X}_{E_{j}}\right|} \cdot D \eta \frac{\nabla \mathcal{X}_{E_{j}}}{\left|\nabla \mathcal{X}_{E_{j}}\right|}\right)\left|\nabla \mathcal{X}_{E_{j}}\right|=\int_{\Omega} \mathcal{X}_{E_{j}} \nabla \cdot\left(u_{j} \eta\right)
$$

for all $\eta \in C_{\mathrm{c}}^{1}\left(\Omega ; \mathbb{R}^{n}\right)$. Assume

$$
\left\|u_{j}\right\|_{\mathrm{H}^{1, \mathrm{p}}(\Omega)}, \int_{\Omega}\left|\nabla \mathcal{X}_{E_{j}}\right| \leqslant \Lambda
$$

for some $\Lambda>0$ and let $u \in \mathrm{H}^{1, p}(\Omega), E \subset \Omega$ and a Radon measure $V$ on $G^{n-1} \Omega$ satisfy

$$
\begin{array}{ll}
u_{j} \rightarrow u & \text { weakly in } \mathrm{H}^{1, \mathrm{p}}(\Omega), \\
\mathcal{X}_{E_{j}} \rightarrow \mathcal{X}_{E} & \text { in } \mathrm{L}^{1}(\Omega), \\
V_{\mu_{j}} \rightarrow V & \text { as varifolds. }
\end{array}
$$

THEOREM A.2 Under the above assumptions we have $V=V_{\mu}$ for an integral $(n-1)$-varifold $\mu$ with locally bounded first variation and mean curvature vector

$$
\vec{H}_{\mu} \in \mathrm{L}_{\mathrm{loc}}^{s}\left(\mu_{V}\right), \quad 1-\frac{n}{p}=-\frac{n-1}{s} .
$$

Moreover $E$ is a set of finite perimeter, $\partial^{*} E \subset \operatorname{spt}(\mu)$, and the mean curvature vector of $\mu$ satisfies

$$
\vec{H}_{\mu}=u v_{E} \quad \mu \text {-almost everywhere, }
$$

where

$$
v_{E}(x)= \begin{cases}\nabla \mathcal{X}_{E}(x) /\left|\nabla \mathcal{X}_{E}\right|(x) & \text { on } \partial^{*} E \\ 0 & \text { otherwise }\end{cases}
$$

\section{REFERENCES}

1. Almgren, F. J. The theory of varifolds. Princeton notes (1965).

2. Almgren, F. Existence and regularity almost everywhere of elliptic variational problems with constraints. Mem. Amer. Math. Soc. 165 (1976). Zbl 0327.49043 MR 54 \#842

3. Ambrosio, L., Fusco, N., \& Pallara, D. Functions of Bounded Variation and Free Discontinuity Problems. Oxford Univ. Press (2000). Zbl 0957.49001 MR 2003a:49002

4. BRAKKe, K. The Motion of a Surface by its Mean Curvature. Princeton Univ. Press (1978). Zbl 0386.53047 MR 82c:49035

5. Evans, L. C. Partial Differential Equations. Graduate Stud. Math. 19, Amer. Math. Soc. (1998). Zbl 0902.35002 
6. Evans, L. C. \& GARIEPY, R. F. Measure Theory and Fine Properties of Functions. CRC Press, Boca Raton (1992). Zbl 0804.28001 MR 93f:28001

7. Federer, H. Geometric Measure Theory. Grundlehren Math. Wiss. 153, Springer (1969). Zbl 0176.00801 MR 41 \#1976

8. GiUsti, E. Minimal Surfaces and Functions of Bounded Variation. Birkhäuser, Boston (1984). Zbl 0545.49018 MR 87a:58041

9. Gurtin, M. E. On the two-phase Stefan problem with interfacial energy and entropy. Arch. Rat. Mech. Anal. 96 (1986), 199-241. Zbl 0654.73008 MR 88j:80001a

10. LuCKhaus, S. Solutions for the two-phase Stefan problem with the Gibbs-Thomson law for the melting temperature. European J. Appl. Math. 1 (1990), 101-111. Zbl 0734.35159 MR 92i:80004

11. Luckhaus, S. The Stefan problem with Gibbs-Thomson law. Preprint, Università di Pisa (1991).

12. LuCKhaus, S. Supplement to [11]: Continuity of $u+\mathcal{X}$. Oral communication.

13. Plotnikov, P. I. \& Starovol̆tov, V. N. The Stefan problem with surface tension as a limit of a phase field model. Differential Equations 29 (1993), 395-404. Zbl 0802.35165 MR 94f:35155

14. Reshetnyak, YU. G. Weak convergence of completely additive vector functions on a set. Siberian Math. J. 9 (1968), 1039-1045. Zbl 0176.44402

15. RÖGER, M. Lösungen für das Stefan Problem mit Gibbs-Thomson Gesetz bei einer lokalen Minimierung. Doctoral thesis, Univ. Bonn (2003).

16. Sсhёtzle, R. A counterexample for an approximation of the Gibbs-Thomson law. Adv. Math. Sci. Appl. 7 (1997), 25-36. Zbl 0883.35043 MR 98c:35161

17. SCHÄTZLE, R. Hypersurfaces with mean curvature given by an ambient Sobolev function. J. Differential Geometry 58 (2001), 371-420. Zbl pre01782693 MR 2003a:49058

18. Schätzle, R. Quadratic tilt-excess decay and strong maximum principle for varifolds. Ann. Scuola Norm. Sup. Pisa Cl. Sci. (to appear).

19. Simon, L. Lectures on Geometric Measure Theory. Proc. Centre Math. Anal. Austral. Nat. Univ. 3 (1983). Zbl 0546.49019 MR 87a:49001

20. Smoller, J. Shock Waves and Reaction-Diffusion Equations. Grundlehren Math. Wiss. 258, Springer, New York (1994). Zbl 0807.35002 MR 95g:35002

21. Tamanini, I. Boundary of Caccioppoli sets with Hölder continuous normal vectors. J. Reine Angew. Math. 334 (1982), 27-39. Zbl 0479.49028 MR 83m:49067

22. Visintin, A. Models of Phase Transition. Birkhäuser, Boston (1996). Zbl 0882.35004 MR 98a:80006 